Preprint DFPD 03/TH/03

February 2003

\title{
Chern-kernels and anomaly cancellation in $M$-theory
}

\author{
K. Lechner ${ }^{1}$ and P.A. Marchetti ${ }^{2}$ \\ Dipartimento di Fisica, Università degli Studi di Padova, \\ and \\ Istituto Nazionale di Fisica Nucleare, Sezione di Padova, \\ Via F. Marzolo, 8, 35131 Padova, Italia
}

\begin{abstract}
This paper deals with magnetic equations of the type $d H=J$ where the current $J$ is a $\delta$-function on a brane worldvolume and $H$ a $p$-form field strength. In many situations in $M$-theory this equation needs to be solved for $H$ in terms of a potential. A standard universality class of solutions, involving Dirac-branes, gives rise to strong intermediate singularities in $H$ which in many physically relevant cases lead to inconsistencies. In this paper we present an alternative universality class of solutions for magnetic equations in terms of Chern-kernels, and provide relevant applications, among which the anomaly-free effective action for open $M 2$-branes ending on M5-branes. The unobservability of the Dirac-brane requires a Dirac quantization condition; we show that the requirement of "unobservability" of the Chern-kernel leads in $M$-theory to classical gravitational anomalies which cancel precisely their quantum counterparts.
\end{abstract}

PACS: 11.15.-q, 11.10.Kk, 11.30.Cp; Keywords: Chern-kernels, branes, anomalies, singular currents.

\footnotetext{
${ }^{1}$ kurt.lechner@pd.infn.it

${ }^{2}$ pieralberto.marchetti@pd.infn.it
} 


\section{Introduction and Summary}

Extended objects represent basic excitations of $M$-theory and string theory; usually they show up in pairs of a brane with electric charge $e$ and a dual brane with magnetic charge $g$; the consistency condition for their coexistence is represented by Dirac's quantization condition

$$
e g=2 \pi n G \text {, }
$$

where $G$ is Newton's constant and $n$ is an integer. In terms of the charges the tensions are given by

$$
T_{e}=e / G, \quad T_{g}=g / G \text {. }
$$

The distinction between branes and dual branes is to a certain extent conventional, we call here (electric) "branes" the lower dimensional objects and (magnetic) "dual branes" the higher dimensional ones. The distinction becomes indeed physical when one considers the couple of classical Maxwell equations arising from supergravity theories with electric and magnetic sources: the electric equation for a "brane"-source gets non-linear corrections, while in most cases the magnetic one for a "dual brane"-source does not and remains of the form

$$
d H=g J_{g},
$$

where $J_{g}$ is the $\delta$-function on the dual brane worldvolume $M_{g}$ and $H$ is the field strength curvature form. In the absence of non-linear corrections the electric Maxwell equation is

$$
d * H=e J_{e},
$$

where $J_{e}$ is the $\delta$-function on the brane worldvolume $M_{e}$. This system of equations is classically consistent if both brane worldvolumes are closed, i.e. $d J_{e}=0=d J_{g}$. If one of the two branes is absent, say the magnetic one, then it is also straightforward to write an action because (1.3) can be solved locally in terms of a potential through $H=d B$, but if both branes are present then the introduction of a potential is more subtle. In this case a standard device to solve the magnetic equation in terms of a potential requires the introduction of a Dirac-brane, i.e. a surface whose boundary is $M_{g}$, and one can write an action which is well defined modulo $2 \pi$ if (1.1) holds. In this case the potential $B$ carries necessarily a singularity along the Dirac-brane, and therefore also on $M_{g}$ which is its boundary. For linear Maxwell equations with sources one can clearly invert the role of branes and dual branes, upon replacing $H \rightarrow * H$ and introducing a dual potential $\widetilde{B}$ instead of $B$.

The present paper deals with situations where the r.h.s. of (1.4) carries non-linear corrections which typically arise in supergravity theories coupled to branes. Examples are $M 5$ - and M2-branes in eleven dimensions, NS5-branes and NS-strings in ten dimensions, and $D$-branes in type $I I$ theories. First of all, due to these corrections, in this case it is not possible to introduce a dual potential $\tilde{B}$ without introducing a potential $B$; this 
means that to write an action one must necessarily solve (1.3) in terms of $B$. Once this has been done one can regard the non-linear version of (1.4) as equation of motion and try to write an action for this equation (so eventually there is no need to introduce $\widetilde{B}$ ).

Since the magnetic equation remained the same as in the linear case a natural attempt to solve it would be again through the introduction of a Dirac-brane. To see if such a procedure works we have to take a closer look on the nature of the non-linear corrections to the r.h.s. of (1.4). In all the examples quoted above these corrections contain terms of the kind

$$
B J_{g} \quad \text { or } \Phi J_{g}
$$

for some target space form $\Phi$, not involving $B^{3}$. In the first term the potential $B$ gets evaluated directly on $M_{g}$; on the other hand, to get a term like $\Phi J_{g}$ in the equation of motion one has to add to the action a term like $\int B \Phi J_{g}=\int_{M_{g}} B \Phi$, and this requires again to evaluate $B$ on $M_{g}$. But, as observed above, in the presence of a Dirac-brane $B$ is singular on $M_{g}$ and hence its restriction to (pullback on) $M_{g}$ is not defined. We conclude that whenever the dynamics of a theory involves a potential solving (1.3) evaluated on the dual brane worldvolume $M_{g}$ itself one has to abandon the Dirac-brane approach for that brane. This seems a dangerous conclusion because the quantization condition (1.1) is intimately related with the use of Dirac-branes.

The main result of this paper is that a way out is provided by Chern-kernels [1]: these are differential forms $K$, satisfying $d K=J_{g}$, generalizing the Coulomb electric field. The fundamental problem with Dirac-branes is that they introduce $\delta$-like singularities along their worldvolume; the forms $K$ instead exhibit milder universal inverse-power-like singularities on $M_{g}$, and they allow to define potentials $B$ which are well-defined on $M_{g}$. They constitute a new universality class of solutions for the magnetic equation (1.3) and they allow to write well-defined effective actions for supergravity theories with electric and/or magnetic sources.

Since the non-linear terms in (1.5) are independent of electric sources, we stress that in the presence of such terms the Chern-kernel approach is needed to write a well-defined action, even if there are only magnetic sources and no electric ones.

Apart from solving a problem which could look rather formal, in relevant situations Chern-kernels lead to classical effective actions which exhibit necessarily gravitational anomalies that cancel in string theory against their quantum counterparts. This can be understood roughly as follows. Chern-kernels are not uniquely defined since the universality class admits infinitely many representatives. Two representatives differ by $K^{\prime}=K+d Q$ and the classical action has to be invariant under such shifts; in other words the Chernkernel has to be "unobservable". To save this invariance one has to add counterterms to the classical action which, in turn, give rise to gravitational anomalies supported on

\footnotetext{
${ }^{3}$ Clearly the term $B J_{g}$ can not appear alone in the equation of motion since it would spoil gauge invariance under $\delta B=d \Lambda$. A complete invariant equation of motion will be given in section five.
} 
$M_{g}$. In all examples we examined these classical anomalies cancel precisely against their quantum counterparts, produced by the chiral fields localized on $M_{g}$.

In principle another way out to solve the above problem, i.e. to keep $B$ free from singularities on the magnetic brane, would be to substitute the $\delta$-like current $J_{g}$ with a regularized smooth one $\widetilde{J}_{g}$, entailing the same total magnetic charge. This procedure has been used in [2, 3] where the gravitational anomaly cancellation for $M 5$-branes in $D=11$ has been discussed for the first time. A problem related with such an approach is that at the quantum level a minimal coupling of a smooth magnetic current to an elementary electric source (i.e. an $M 2$-brane with $\delta$-like support) spoils the unobservability of the electric Dirac-brane. This point will be clarified in the next section. The authors of reference 4] performed a redefinition of the three-form potential $B$ used in [2, which allows to remove the regularization. In this adapted version, which has been further applied to a five-dimensional $M 5$-brane toy-model in [5], the approach of [2, 3, 4] becomes indeed comparable with the Chern-kernel approach. The relevant differences will be discussed at the end of section five.

Self-dual branes play a special role and exhibit an additional peculiar feature with respect to a system of branes/dual-branes. By definition a self-dual brane is a brane minimally coupled to a self-dual field strength in a $(4 N+2)$-dimensional space-time. This means that even in the absence of non-linear terms the minimal coupling $\int_{M} B$ becomes problematic if $B$ has Dirac-brane-type singularities along $M$. As we will show, in this case in principle both possibilities - a Dirac-brane (with a framing regularization) or a Chern-kernel - are allowed, but they are physically inequivalent: in the Dirac-brane approach the dynamics is consistent if the charge is quantized, while in the Chern-kernel approach there is no need of charge quantization, but a (classical) gravitational anomaly shows up, which for consistency should be canceled by a quantum counterpart. Eventually for self-dual branes it is the particular physical situation that decides which approach one has to use.

In section two we review briefly the Dirac-brane approach, indicating to which extent it can be circumvented in compatibility with (1.1). In section three we define Chern-kernels for even and odd ranks and state their main properties, while section four is devoted to self-dual branes. In section five we illustrate how Chern-kernels work in a highly non trivial case: $M 2$-branes ending on $M 5$-branes. This is actually a more general case then the one we referred to above since the electric current is not conserved and there are quantum gravitational anomalies not only on the (magnetic) M5-brane, but also on the boundary of $M 2$; this boundary corresponds indeed to a self-dual string. Actually, in this case anomaly cancellation has been realized until now only partially [6]. Section six gives a further application: a $B_{3} \leftrightarrow \widetilde{B}_{6}$-duality symmetric action for bosonic $D=11$ supergravity with electric and magnetic sources. Section seven is devoted to concluding remarks.

The present paper provides also some proofs which, for lack of space, have not been 
reported in [7].

A comment on our mathematical framework is in order. Since our currents $J$ carry a $\delta$-like support they are not smooth differential $p$-forms, they are rather distribution valued so called $p$-currents [8]. Consistency then requires that all our differential forms have to be considered in the space of distributions and that also the differential operator $d$ acts in the sense of distributions. This implies that we have always

$$
d^{2}=0,
$$

and that in a topologically trivial space-time a closed form is always exact. In this framework two caveats are in order: first, the product of two distributions is in general not a distribution if the singularities involved are too strong; second, Leibntz's rule does in general not hold: for even forms the relation $d(\phi \psi)=\phi d \psi+d \phi \psi$ may not be valid, even if the product $\phi \psi$ is well-defined (a trivial example is $\phi=\psi=\varepsilon(x)$, the sign function). As we will see, in the Chern-kernel approach all our products will amount to well-defined distributions, and we will have to worry only about Leibnitz's rule. Henceforth, according to the standard physical terminology, we will call the $p$-currents again $p$-forms.

In most of the paper we assume to be in a topologically trivial space-time, some topological aspects will be discussed in the concluding section.

\section{Dirac-branes}

We review here briefly the standard Dirac-brane approach, allowing to write an action for the linear system (1.3), (1.4). $J_{g}$ is the $\delta$-function on the closed worldvolume $M_{g}$, more precisely it is the "Poincarè-dual in the space of $p$-currents" of $M_{g}$ : such forms are called integer forms. For a precise definition and basic properties of this Poincarè-duality see e.g. [8, 9]. Here we recall that by definition the Poincarè-dual $J_{M}$ of a manifold $M$ satisfies

$$
\int \Phi J_{M}=\int_{M} \Phi
$$

for every test form $\Phi$. The integral of products of integer forms, closed or not, is always integer whenever it is well defined. The operator $d$ corresponds to the boundary operator $\partial$ on manifolds. If multiplied by a target-space form, $J_{M}$ performs the pullback on $M$ :

$$
\Phi J_{M}=\left.\Phi\right|_{M} J_{M}
$$

Let $M_{g}$ be a closed $d$-dimensional manifold in a $D$-dimensional space-time. Since $M_{g}$ is closed we can introduce a $(d+1)$-surface $S_{g}$ - a Dirac-brane - whose boundary is $M_{g}$, $M_{g}=\partial S_{g}$. The Dirac-brane is clearly not unique: choosing another Dirac-brane we have $M_{g}=\partial S_{g}^{\prime}$ and there exists an interpolating $(d+2)$-surface $T_{g}$ such that $S_{g}^{\prime}-S_{g}=\partial T_{g}$. The corresponding relations for the Poincarè-duals of these surfaces are

$$
J_{g}=d C_{g}=d C_{g}^{\prime}, \quad C_{g}^{\prime}-C_{g}=d W_{g},
$$


where $J_{g}, C_{g}$ and $W_{g}$ are integer forms of rank $(D-d),(D-d-1)$ and $(D-d-2)$ respectively.

The first relation of (2.1) permits to solve (1.3) in terms of a potential $B$

$$
H=d B+g C_{g}
$$

and to write then an action giving rise to (1.4):

$$
I_{1}=\frac{1}{G} \int\left(\frac{1}{2} H * H-e B J_{e}\right) \equiv I_{k i n}+I_{w z}
$$

where according to string theory for the overall normalization we used Newton's constant.

To keep the field strength invariant under a change of Dirac-brane we have to require that $B$ transforms as

$$
B^{\prime}=B-g W_{g}
$$

where, we recall, $W_{g}$ is the $\delta$-function on the surface $T_{g}$. Under this transformation $I_{k i n}$ is manifestly invariant while the Wess-Zumino term changes as

$$
\Delta I_{w z}=\frac{e g}{G} \int W_{g} J_{e}
$$

Since the integrand is a product of integer forms, the integral is integer and $\Delta I_{w z}$ is an integer multiple of $2 \pi$ thanks to charge quantization (1.1). From (2.4) we retrieve that the potential $B$ is ill-defined on $M_{g}$ because the form $W_{g}$ is the $\delta$-function on the surface $T_{g}$ which, by construction, contains as submanifold $M_{g}$; so $W_{g}$ does not admit pullback on $M_{g}$ and due to (2.4) $B$ bears the same fate.

This is in synthesis the standard Dirac-brane construction of an action for Maxwell equations in the presence of electric and magnetic sources. Let us now see how stringent it is. First we note that one can introduce also an arbitrary antiderivative $K_{e}$ for the conserved electric current, $J_{e}=d K_{e}$, and rewrite the Wess-Zumino term as

$$
I_{w z}=\frac{e}{G} \int d B K_{e}=\frac{e}{G} \int H K_{e}-\frac{e g}{G} \int C_{g} K_{e}
$$

So in the Dirac-brane approach, as it stands, one has really an asymmetric treatment for electric and magnetic sources: for the magnetic source one introduces a Dirac-brane as antiderivative, while for the electric one one can introduce an arbitrary antiderivative; but due to duality one expects that the situation can be reversed. This is indeed the case. Choose for $K_{e}$, which is arbitrary, the Poincarè-dual $C_{e}$ of an electric Dirac-brane, $J_{e}=d C_{e}$. Then the integrand in the last term in (2.6) is a product of integer forms, and the term itself becomes an integer multiple of $2 \pi$ due to charge quantization; it can therefore be disregarded. The action $I_{1}$ can then be rewritten as

$$
I_{2}=\frac{1}{G} \int\left(\frac{1}{2} H * H+e H C_{e}\right),
$$


where the magnetic Dirac-brane does now no longer appear explicitly. This means that in this form of the action one can take

$$
H=d B+g K_{g}
$$

with $K_{g}$ an arbitrary antiderivative of the magnetic current,

$$
J_{g}=d K_{g}
$$

As $I_{1}$ had a spurious dependence on the magnetic Dirac-brane, the action $I_{2}$ has now a spurious dependence on the electric Dirac-brane; for $C_{e}^{\prime}=C_{e}+d W_{e}$, with $W_{e}$ the $\delta$-function on a suitable surface, $I_{2}$ changes by

$$
\Delta I_{2}=\frac{e}{G} \int H d W_{e}=-\frac{e g}{G} \int J_{g} W_{e},
$$

again an integer multiple of $2 \pi$. It is obvious that $I_{2}$ gives as equation of motion still (1.4). So the situation is now indeed reversed: with the price of introducing an (unobservable) electric Dirac-brane, we can choose for the magnetic brane an arbitrary form $K_{g}$ satisfying (2.9).

With this simple observation we have now a new possibility for introducing a potential. The main difference between (2.2) and (2.8) is the following. We have $d K_{g}=J_{g}=d C_{g}$, and this means that $C_{g}$ as well as $K_{g}$ are necessarily singular on the magnetic brane because $J_{g}$ is the $\delta$-function on $M_{g}$. $C_{g}$ has $\delta$-function like singularities along a Diracbrane, say along a fixed space-time direction; these singularities are not universal since there is no preferred direction in space-time: changing the Dirac-brane changes this space-time direction and hence the support of the singularities, but this means that $B$ itself has to change by singular terms - see (2.4) - interpolating between the two different singular behaviours, see also [9, 10]. Consider now the second possibility (2.8). In this case $K_{g}$ has to satisfy a priori only $d K_{g}=J_{g}$ with no further restrictions; but then, as we know from the Coulomb electric field whose divergence is a $\delta$-function, its singularities can be also of the inverse-power-type, i.e. milder then $\delta$-type singularities: this behaviour is indeed realized by a Chern-kernel. The problem with inverse-power-like singularities is that, as in the Dirac-brane case, a priori they can still change when $K_{g}$ changes by a closed form. This problem will be solved in the next section.

From (2.5) and (2.10) it is also clear that magnetic and electric branes can be consistently minimally coupled at the quantum level only if both currents carry a $\delta$-like support i.e. if both are integer forms, otherwise either $W_{e}\left(W_{g}\right)$ or $J_{g}\left(J_{e}\right)$ would not be an integer form and the integrals appearing in (2.5), (2.10) would not be integer: as consequence under a change of Dirac-brane the action would not change by an integer multiple of $2 \pi$, even if the charges are quantized. 


\section{Chern-kernels}

Chern-kernels are differential forms $K$ satisfying

$$
J=d K,
$$

with a specific singular behaviour near the brane. We take $J$ as an $n$-form whose Poincarèdual is a closed $(D-n)$-dimensional brane worldvolume $M$ in a $D$-dimensional spacetime; the Chern-kernel is then an $(n-1)$-form. The main motivation for an analysis of its properties is that its defining relation allows to solve the magnetic equation $d H=g J$ as

$$
H=d B+g K
$$

\subsection{Brane geometry}

We introduce first the main geometrical quantities which are defined on the brane worldvolume $M$. The brane is parametrized locally by $x^{\mu}(\sigma), \mu=(0, \cdots, D-1)$, and carries a $(D-n)$-dimensional tangent space spanned by $E_{i}^{\mu}(\sigma) \equiv \partial_{i} x^{\mu}(\sigma),(i=0, \cdots, D-n-1)$. The normal $S O(n)$-fiber is spanned by the vectors $N_{\mu}^{a}(\sigma)$ satisfying

$$
N_{\mu}^{a} E_{i}^{\mu}=0, \quad N_{\mu}^{a} N^{\mu b}=\delta^{a b},
$$

where $a=(1, \cdots, n)$. These basis vectors for the normal space are defined modulo a local $S O(n)$-rotation,

$$
N_{\mu}^{\prime a}(\sigma)=\Lambda^{a b}(\sigma) N_{\mu}^{b}(\sigma) .
$$

On $M$ we can introduce also an $S O(n)$-connection $a^{a b}(\sigma)^{4}$, and its curvature $f^{a b}=$ $d a^{a b}+a^{a c} a^{c b}$. For later purposes it is convenient to extend $a$ to an $S O(n)$-connection $A(x)$ on the whole space-time, that is asymptotically flat along directions orthogonal to the brane. We call the corresponding curvature $F \equiv d A+A A$,

$$
\left.A\right|_{M}=a,\left.\quad F\right|_{M}=f
$$

where $\left.\right|_{M}$ means pullback of a space-time form on $M$. While $a$ and $f$ are physical data, the quantities $A$ and $F$ are not: so when they are used at intermediate steps, eventually one has to show that physics is independent of these unphysical data. This means that one can look at $A$ and $F$ as "holographic extensions" of $a$ and $f$ from $M$ to the whole space-time, which do not introduce new degrees of freedom.

To $M$ we can associate also systems of normal coordinates. Such a system realizes a diffeomorphism from the coordinates $x^{\mu}$ to the coordinates $\left(\sigma^{i}, y^{a}\right)$, with $i=0, \cdots, D-$

\footnotetext{
${ }^{4}$ This connection can be parametrized in terms of the normal vectors by $a^{a b}=N^{\mu b}\left(d N_{\mu}^{a}+\Gamma_{\mu}^{\nu} N_{\nu}^{a}\right)$, where $\Gamma_{\mu}^{\nu}$ is the pullback on $M$ of the affine space-time connection. We suppose to work in a curved space-time, so the indices $\mu, \nu, \ldots$ are raised and lowered with the metric $g_{\mu \nu}$ evaluated, in case, on $M$.
} 
$n-1$ and $a=1, \cdots, n$, specified by the functions $x^{\mu}(\sigma, y)$. As a power series in $y-$ the normal coordinates - one has

$$
x^{\mu}(\sigma, y)=x^{\mu}(\sigma)+y^{a} N^{\mu a}(\sigma)+o\left(y^{2}\right) .
$$

Inversion of the diffeomorphism leads to the space-time functions $y^{a}(x)$ with

$$
\left.y^{a}\right|_{M}=0,\left.\quad \partial_{\mu} y^{a}\right|_{M}=N_{\mu}^{a} .
$$

Throughout this paper we suppose that the functions $y^{a}(x)$, with this behaviour on $M$, are globally defined; for more general situations see [7] and section seven. Again, the physical content of these functions is only their behaviour (3.7) on $M$, their values away from $M$ correspond to unphysical data which eventually have to be unobservable.

Actually, the behaviour (3.7) on $M$ is defined up to an $S O(n)$-transformation, as are the normal coordinates themselves. Formally, through the functions $A^{a b}(x)$ and $y^{a}(x)$, we have thus extended the $S O(n)$-structure to the whole space-time. In particular we can define the $S O(n)$-covariant derivatives $D y^{a}=d y^{a}+y^{b} A^{b a}$.

For future purposes it is convenient to introduce an additional (equivalent) $S O(n)-$ connection $\mathcal{A}$ and related curvature $\mathcal{F}$. Set

$$
\hat{y}^{a}=y^{a} / y, \quad \hat{y}^{a} \hat{y}^{a}=1
$$

and define

$$
\mathcal{A}^{a b}=A^{a b}-2 \hat{y}^{[a} D \hat{y}^{b]} \equiv A^{a b}+X^{a b} .
$$

This gives for the curvature

$$
\mathcal{F}^{a b}=d \mathcal{A}^{a b}+\mathcal{A}^{a c} \mathcal{A}^{c b}=F^{a b}+D \hat{y}^{a} D \hat{y}^{b}+2 \hat{y}^{[a} F^{b] c} \hat{y}^{c} .
$$

The qualifying properties of this connection are that its curvature has vanishing components along $\hat{y}^{a}$ and that $\hat{y}^{a}$ is covariantly constant with respect to it,

$$
\hat{y}^{a} \mathcal{F}^{a b}=0, \quad D(\mathcal{A}) \hat{y}^{a} \equiv d \hat{y}^{a}+\hat{y}^{b} \mathcal{A}^{b a}=0 .
$$

Notice that, contrary to $A$ and $F$, the forms $\mathcal{A}$ and $\mathcal{F}$ do not admit pullback on $M$.

\subsection{The Coulomb form}

In terms of normal coordinates the current can be expressed in an $S O(n)$-invariant way as

$$
J=\frac{1}{n !} \varepsilon^{a_{1} \cdots a_{n}} d y^{a_{1}} \cdots d y^{a_{n}} \delta^{n}(y),
$$

signaling of course that the brane stays at $y=0$.

In searching for an $(n-1)$-form whose differential equals $J$ a first attempt would be to consider a generalization of the three-dimensional Coulomb electric field, more precisely of its Hodge-dual

$$
K_{0}=\frac{1}{8 \pi} \varepsilon^{a b c} \frac{1}{y^{3}} y^{a} d y^{b} d y^{c}=\frac{1}{8 \pi} \varepsilon^{a b c} \hat{y}^{a} d \hat{y}^{b} d \hat{y}^{c}
$$


corresponding to $n=3$. This formula can also be regarded as the angular form on a two-sphere, with unit integral. Its generalization for a generic $n$ is:

$$
K_{0}=\frac{(-)^{n+1} \Gamma(n / 2)}{2 \pi^{n / 2}(n-1) !} \varepsilon^{a_{1} \cdots a_{n}} \hat{y}^{a_{1}} d \hat{y}^{a_{2}} \cdots d \hat{y}^{a_{n}},
$$

and it is easy to see that indeed

$$
d K_{0}=J
$$

The problem with the Coulomb form in (3.11) is that its (singular) behaviour on $M$, i.e. as $y \rightarrow 0$, is not universal but depends on the particular normal coordinate functions $y^{a}(x)$ we have chosen. In other words, it is invariant under global $S O(n)$-rotations, but not under local ones: for different normal coordinates one has indeed $\hat{y}^{\prime a}=\Lambda^{a b} \hat{y}^{b}$, where $\Lambda^{a b}(x)$ is an $S O(n)$-matrix. For the Coulomb form associated to the rotated normal coordinates we have of course still $d K_{0}^{\prime}=J$, and hence

$$
K_{0}^{\prime}-K_{0}=d Q_{0}
$$

but the form $Q_{0}$ is singular on $M$, because the matrix $\Lambda^{a b}(x)$ does not reduce to the identity on $M$, meaning that $K_{0}$ and $K_{0}^{\prime}$ exhibit different singularities.

The question is then if $K_{0}$ admits a completion $K$ carrying an $S O(n)$-invariant singular behaviour on $M$, which solves still the magnetic equation (3.12). Clearly one should then have

$$
K=K_{0}+d \Phi
$$

for a convenient form $\Phi$. The answer to this question is affirmative, but since it entails completely different features for even and odd currents we treat the two cases separately. The difference originates essentially from the fact that the Euler characteristics of an odd bundle is zero.

\subsection{Even Chern-kernels}

For $n$ odd the Coulomb-form can be completed to an $S O(n)$-invariant Chern-kernel given by 11

$$
K=\frac{\Gamma(n / 2)}{2 \pi^{n / 2}(n-1) !} \varepsilon^{a_{1} \cdots a_{n}} \hat{y}^{a_{1}} \mathcal{F}^{a_{2} a_{3}} \ldots \mathcal{F}^{a_{n-1} a_{n}},
$$

where $\mathcal{F}^{a b}$, see equation (3.9), reduces here actually to $F^{a b}+D \hat{y}^{a} D \hat{y}^{b}$, due to the presence of the factor $\hat{y}^{a_{1}}$. SO $(n)$-invariance is manifest, and one has only to show that $d K=J$. Consider first the $\delta$-function contribution to $d K$. It is immediately seen that $K$ contains as building block $K_{0}$ which, for dimensional reasons, is the unique term which can give rise to a distribution-valued contribution, and we know already that $d K_{0}=J$. It remains to show that formally, i.e. neglecting the $\delta$-function contribution, $K$ is closed. This is obvious if one observes that, since $K$ is invariant, the differential $d$ can be substituted 
with the differential covariant w.r.t. $\mathcal{A}, d K=D(\mathcal{A}) K$. The conclusion follows then from the second relation in (3.10) and from $D(\mathcal{A}) \mathcal{F}=0$.

Since the expression for $K$ is a polynomial function of $\hat{y}$ and $A$ it is also clear that (3.13) holds with $\Phi$ polynomial in $\hat{y}$ and $A$ as well. For $n=3$ one has e.g.

$$
K=\frac{1}{8 \pi} \varepsilon^{a b c} \hat{y}^{a}\left(F^{b c}+D \hat{y}^{b} D \hat{y}^{c}\right), \quad \Phi=\frac{1}{8 \pi} \varepsilon^{a b c} \hat{y}^{a} A^{b c}
$$

With respect to $K_{0}$, which carries $n$ powers of (the singular functions) $\hat{y}$, the even Chernkernel contains additional subleading singular terms with powers of $\hat{y}$ ranging from 1 to $n-2$ : these subleading terms are required to form an invariant singular behaviour.

As it stands $K$ depends on the data $A^{a b}(x)$ and $y^{a}(x)$, whose behaviour away from $M$ is unphysical. The dependence on these data is in some sense analogous to the dependence on the Dirac-brane in the Dirac-brane approach, and it must be compensated by a transformation of the potential $B$, see (3.2). Changes in these data $A \rightarrow A^{\prime}, y \rightarrow y^{\prime}$ are constrained by the boundary values on $M$, (3.5) and (3.7), where they must reduce to an $S O(n)$-rotation. Since we have in any case $\hat{y}^{\prime a} \hat{y}^{\prime a}=1$, the most general changes can be parametrized by

$$
\hat{y}^{\prime}=\Lambda \hat{y}, \quad A^{\prime}=\Lambda(A+W) \Lambda^{T}-\Lambda d \Lambda^{T},
$$

where $\Lambda(x) \in S O(n)$ and the arbitrary Lie-algebra valued one-form $W$ is constrained only to vanish on $M$,

$$
\left.W\right|_{M}=0 \text {. }
$$

Consider now the relation between two different Chern-kernels; since the differential of a Chern-kernel equals always $J$ it is clear that the difference is an exact form,

$$
K^{\prime}=K+d Q
$$

But (3.16) implies that moreover

$$
\left.Q\right|_{M}=0
$$

Indeed, due to $S O(n)$-invariance

$$
K^{\prime}-K=K\left(A^{\prime}, \hat{y}^{\prime}\right)-K(A, \hat{y})=K(A+W, \hat{y})-K(A, \hat{y})
$$

and since $K_{0}$ is independent of $A(3.13)$ gives

$$
Q=\Phi(A+W, \hat{y})-\Phi(A, \hat{y})
$$

which contains at least one power of $W$. For $n=3$ one has

$$
Q=\frac{1}{8 \pi} \varepsilon^{a b c} \hat{y}^{a} W^{b c} .
$$

Equation (3.16) implies then (3.18). 
This shows not only that the even Chern-kernel has a universal singular behaviour on $M$ (for that it would be sufficient to show that $\left.Q\right|_{M}$ is a well defined form), but also that the pullback of the potential $B$ is a completely invariant form: since invariance of the field strength in (3.2) demands

$$
B^{\prime}=B-g Q
$$

one gets

$$
\left.B^{\prime}\right|_{M}=\left.B\right|_{M}
$$

We call the transformations (3.17), (3.19) Q-transformations; the dynamics of a physical theory must be independent of the particular Chern-kernel used as antiderivative of $J$, meaning that its action has to be invariant under such transformations. $Q$-invariance will therefore be a guiding principle for constructing consistent actions for magnetic branes with non-linear interactions; in the presence of closed electric branes we must, in addition, require independence of the action of the electric Dirac-brane. In this sense $Q$-invariance plays a role similar to unobservability of a Dirac-brane: the transformations (2.1) and (2.4) coincide indeed formally with (3.17) and (3.19).

We emphasize that in this framework the physical gravitational normal bundle $S O(n)-$ transformations on $M$ arise as $Q$-transformations restricted to $M$, with transformation parameter $\lambda=\left.\Lambda\right|_{M}$, see (3.15).

Since even Chern-kernels have an invariant behaviour near the brane and lead, therefore, to potentials which are invariant when evaluated on the brane, one might think that even Chern-kernels have nothing to do with gravitational anomalies localized on the brane; this is however not the case since products of an even number of Chern-kernels are cohomologically equivalent to characteristic classes, i.e. to invariant polynomials of the normal bundle $S O(n)$-curvature $F$ which, upon descent, give rise to gravitational anomalies. An important example in which the even Chern-kernel-approach leads to cancellation of the quantum normal bundle anomaly is represented by the $M 5$-brane, see [7. For this reason we discuss in the next subsection the basic properties of such products.

\subsection{Products of even Chern-kernels}

In this section we derive algebraic relations involving powers of the even Chern-kernel, which are needed in the construction of effective actions for $p$-branes in $M$-theory, see section five.

In general the product of two distributions does not define a distribution, but in the case of even Chern-kernels the product $K \ldots K$ defines still a distribution-valued differential form, with again inverse-power-like singularities on $M$. Contrary to $K$, however, the even powers such as $K K$ are closed forms. This is not in contrast with $d K=J$ since what fails here is Leibnitz's rule: the formal computation $d(K K)=2 K J$ makes indeed no sense because $K$ does not admit pullback on $M$; we must first evaluate the product and then 
take the differential. We will actually now show that there exists a form $S$, polynomial in $A$ and $\hat{y}$ but not $S O(n)$-invariant, such that (see also [12, 13])

$$
K K=\frac{1}{4} d S
$$

We begin with the evaluation of $K K$. Recalling that $\hat{y}^{a} \mathcal{F}^{a b}=0$, from the definition (3.14) this product is easily seen to reduce to a combination of traces of $\mathcal{F}$. Setting $n=2 m+1$ one obtains

$$
K K=\frac{1}{4} P(\mathcal{F})
$$

where $P$ is the $m$-th Pontrjagin form. Remembering (3.8), a standard transgression formula gives then

$$
P(\mathcal{F})=P(F)+d Y
$$

where $Y$ is an $S O(n)$-invariant $(2 n-3)$-form, polynomial in $\hat{y}, D \hat{y}$ and $F$, singular on $M$ :

$$
Y=2 m \int_{0}^{1} P\left(F_{t}, \cdots, F_{t}, X\right) d t
$$

where $A_{t}=A+t X$ and $F_{t}=d A_{t}+A_{t} A_{t}$. This leads to ${ }^{5}$

$$
S=P^{0}(A)+Y
$$

where $P^{0}(A)$ is the Chern-Simons form associated to $P(F)$. For generic characteristic classes we use the descent notation

$$
d P=0, \quad P=d P^{0}, \quad \delta P^{0}=d P^{1} .
$$

The form $S$ is made out of two contributions: $P^{0}(A)$ is non-invariant but regular on $M$, while $Y$ is invariant but singular on $M$, due to the presence of the singular form $X$. For $n=3$ one has

$$
\begin{aligned}
P(F) & =-\frac{1}{2(2 \pi)^{2}} \operatorname{tr} F^{2}, \\
P^{0}(A) & =-\frac{1}{2(2 \pi)^{2}} \operatorname{tr}\left(A d A+\frac{2}{3} A^{3}\right), \\
Y & =-\frac{1}{(2 \pi)^{2}} \hat{y}^{a} D \hat{y}^{b} F^{a b} .
\end{aligned}
$$

From the formulae above it is clear that $K K$ is a closed form. What happens is essentially that $K$ contains only odd powers of $\hat{y}$ 's - in particular the Coulomb form whose differential gives rise to $J$ - while $K K$ contains only even powers of $\hat{y}$ 's and so no $\delta$-function contributions can show up in its differential.

\footnotetext{
${ }^{5} S$ differs from the canonical Chern-Simons form $P^{0}(\mathcal{A})$, associated to $P(\mathcal{F})=d P^{0}(\mathcal{A})$, by a closed form.
} 
In considering the triple product $K K K$ one encounters the form $d Y K$ which can be seen to be closed too, more precisely one has

$$
d(Y K)=d Y K
$$

The key point is again to see if the product $Y K$ contains terms whose differential can give rise to $\delta$-function contributions ${ }^{6}$. Such terms must contain precisely $n-1$ powers of $d \hat{y}$, as $K_{0}$. In this case we observe that $K(Y)$, being invariant, contains only even (odd) powers of $D \hat{y}$; this means that $Y K$ contains only odd powers of $D \hat{y}$, with maximum power $n-2$ (because the product of $n$ or more of them is zero by antisymmetry). So the maximum power of $d \hat{y}$ showing up in $Y K$ is $n-2$, and no Coulomb form can appear. This means that one can compute the differential of $Y K$ algebraically, i.e. ignoring $\delta$-function contributions, and (3.24) follows.

Since $P(F)$ is regular on $M$ we can summarize these properties as

$$
d K=J, \quad d(K K)=0, \quad d(K K K)=\frac{1}{4} P(F) J
$$

\subsection{Odd Chern-kernels}

In this case the current $J$ is an even form, $n=2 m$, and a construction like (3.14) is no longer available. On the other hand, the normal bundle of the brane is now even and one can define an $S O(n)$-Euler form $\chi(F)$, and the associated Chern-Simons form $\chi^{0}(A)$, in a standard way:

$$
\chi(F)=\frac{1}{m !(4 \pi)^{m}} \varepsilon^{a_{1} \cdots a_{n}} F^{a_{1} a_{2}} \cdots F^{a_{n-1} a_{n}} \equiv d \chi^{0}(A) .
$$

We will now show that the odd Chern-kernel can be written as a sum ${ }^{7}$

$$
K=\Omega+\chi^{0}(A)
$$

where $\Omega$ is the $S O(n)$-invariant $(n-1)$-form, polynomial in $\hat{y}, D \hat{y}$ and $F$, given in (3.30). So the main difference between even and odd Chern-kernels is that the former are $S O(n)-$ invariant forms, while the latter are not, due to the presence of the Chern-Simons form.

To prove that the expression in (3.27) satisfies $d K=J$, and to find an explicit expression for $\Omega$ we start from the observation that the Euler form of the curvature $\mathcal{F}$ vanishes identically,

$$
\chi(\mathcal{F})=0 .
$$

This is a direct consequence of $\hat{y}^{a} \mathcal{F}^{a b}=0^{8}$. On the other hand, the shift-relation (3.8) allows to express the Euler form for $\mathcal{F}$ in terms of the Euler form for $F$,

$$
\chi(\mathcal{F})=\chi(F)+d \Omega-J,
$$

\footnotetext{
${ }^{6}$ For an alternative proof see appendix A.

${ }^{7}$ Strictly speaking, the "odd spherical kernel" introduced by Chern 1$]$ is $\Omega$.

${ }^{8}$ More concretely, take the identity $\hat{y}^{[b} \mathcal{F}^{a_{1} a_{2}} \ldots \mathcal{F}^{\left.a_{n-1} a_{n}\right]}=0$, which holds because one has $n+1$ antisymmetric indices, and contract it with $\hat{y}^{b}$.
} 
where, defining as above $A_{t}=A+t X$ and $F_{t}=d A_{t}+A_{t} A_{t}$, according to standard transgression one has

$$
\Omega=m \int_{0}^{1} \chi\left(F_{t}, \cdots, F_{t}, X\right) d t .
$$

This explains the algebraic contributions in (3.29); the subtraction of $J$ is necessary because by direct inspection one can see that $\Omega$ contains as top form in $d \hat{y}$ precisely the Coulomb form $K_{0}$, and since our differential acts in the sense of distributions this implies that $d \Omega$ contains as $\delta$-function contribution precisely $J$, which has to be subtracted. The identities (3.28) and (3.29) ensure then that $d K=J$.

The evaluation of the transgression formula (3.30) is straightforward, for $n=4$ one obtains e.g.

$$
\begin{aligned}
\Omega & =-\frac{1}{2(4 \pi)^{2}} \varepsilon^{a_{1} \ldots a_{4}} \hat{y}^{a_{1}} D \hat{y}^{a_{2}}\left(4 F^{a_{3} a_{4}}+\frac{8}{3} D \hat{y}^{a_{3}} D \hat{y}^{a_{4}}\right), \\
\chi^{0}(A) & =\frac{1}{2(4 \pi)^{2}} \varepsilon^{a_{1} \ldots a_{4}}\left(A^{a_{1} a_{2}} d A^{a_{3} a_{4}}+\frac{2}{3} A^{a_{1} a_{2}}(A A)^{a_{3} a_{4}}\right), \\
\Phi & =-\frac{1}{2(4 \pi)^{2}} \varepsilon^{a_{1} \ldots a_{4}} \hat{y}^{a_{1}}\left(4 d \hat{y}^{a_{2}}+2 \hat{y}^{b} A^{b a_{2}}\right) A^{a_{3} a_{4}},
\end{aligned}
$$

where the form $\Phi$ refers to the decomposition (3.13) which holds clearly also for odd kernels. Notice in particular in $\Omega$ the presence of the Coulomb form $K_{0}$.

Also the odd kernel is subject to $Q$-transformations, i.e. to the changes $y \rightarrow y^{\prime}$, $A \rightarrow A^{\prime}$ as the even one (see (3.15)) and we have also here

$$
K^{\prime}=K+d Q, \quad B^{\prime}=B-g Q
$$

for some $Q$. This time one gets

$$
K\left(A^{\prime}, \hat{y}^{\prime}\right)-K(A, \hat{y})=K(A+W, \hat{y})-K(A, \hat{y})+\chi^{0}\left(A^{\prime}\right)-\chi^{0}(A+W)
$$

Since the Chern-Simons forms differ by the $S O(n)$-rotation $\Lambda$ we have

$$
\chi^{0}\left(A^{\prime}\right)-\chi^{0}(A+W)=d \chi^{1}(A+W)
$$

leading to

$$
Q=\Phi(A+W, \hat{y})-\Phi(A, \hat{y})+\chi^{1}(A+W)
$$

where in $\chi^{1}$ we suppressed the dependence on $\Lambda$. The pullback of $Q$ on $M$ is then again finite, but now different from zero. Since $\left.W\right|_{M}=0$ one gets

$$
\left.Q\right|_{M}=\chi^{1}(a)
$$

where, we recall, $a$ is the (physical) $S O(n)$-connection on $M, a=\left.A\right|_{M}$. For an infinitesimal transformation the form $\chi^{1}(a)$ is really the descent of the Euler form on $M$,

$$
\chi(f)=d \chi^{0}(a), \quad \delta \chi^{0}(a)=d \chi^{1}(a) .
$$


For the pullback of the potential we obtain then the anomalous transformation law

$$
\left.B^{\prime}\right|_{M}=\left.B\right|_{M}-g \chi^{1}(a) .
$$

We can summarize the results of this section as follows. The odd Chern-kernel is made out of two terms, one is singular on $M$ and the other is regular. The singular contribution $\Omega$ is invariant and encodes, therefore, the singularities of $K$ in a universal way. Vice versa, the regular contribution $\chi^{0}(A)$ transforms non trivially under $S O(n)$. For the potential $B$ this implies that it admits a finite pullback $\left.B\right|_{M}$ that under $Q$-transformations undergoes an anomalous $S O(n)$-transformation.

It is clear that the anomalous transformation law for $\left.B\right|_{M}$ plays a role in gravitational anomaly cancellation in $M$-theory; for a basic examplification of this feature - for $n=4-$ in the case of the anomalies of the $N S 5$-brane in $D=10, I I A$-supergravity, see reference [14. In the next section we illustrate the occurrence of odd Chern-kernels in a further important case: self-dual branes.

For computational reasons sometimes it may be useful, though not strictly necessary, to have at ones disposal regularized currents and associated regularized Chern-kernels, i.e. a family of forms $K^{\varepsilon}$ and $J^{\varepsilon}$ with $J^{\varepsilon}=d K^{\varepsilon}$, which are regular at $M$ for any $\varepsilon>0$, such that in the sense of distributions

$$
\lim _{\varepsilon \rightarrow 0} J^{\varepsilon}=J, \quad \lim _{\varepsilon \rightarrow 0} K^{\varepsilon}=K
$$

In appendix A we present a particular class of such regularizations, which goes under the name of "real algebraic approximation mode" [11, that keeps the currents and the even Chern-kernels invariant, and that preserves the transformation properties of the odd kernels. Such regularized objects are useful for example in determining the singularity structures of products involving Chern-kernels and currents.

\section{Self-dual branes and chiral bosons}

Self-dual branes are closed branes with a $2 N$-dimensional worldvolume $M$, coupling in a $(4 N+2)$-dimensional space-time minimally to a chiral boson. More precisely we have the equations

$$
\begin{aligned}
d H & =g J \\
H & =* H,
\end{aligned}
$$

where the current $J$ is a $(2 N+2)$-form, the $\delta$-function on $M$. The magnetic and electric Maxwell equations are thus identified.

In writing an action for this system one has to face two problems; the first regards the construction of a covariant action for the chiral boson, a problem which is elegantly solved 
by the PST-approach [15]. The second problem regards the introduction of a potential $B$. Formally the PST-approach furnishes the action

$$
I=\frac{1}{2 G} \int\left(H * H-\mathcal{H}_{-} * \mathcal{H}_{-}\right)-\frac{g}{G} \int_{M} B
$$

where $\mathcal{H}_{-}=i_{v}(H-* H)$, and $v$ is the auxiliary non-propagating PST-vector. This action leads to the equation of motion $H=* H$, once one has solved the magnetic equation (4.1) in terms of a potential. In principle we have now two ways for doing so.

1) Dirac-branes. We can introduce a Dirac-brane for $M$, with Poincaré-dual $C$, and write $J=d C$. This gives

$$
H=d B+g C
$$

and $B$ has the known singularities on $M$. Consequently the Wess-Zumino term in the action $\int_{M} B=\int B J$ is ill-defined, even in this elementary case with only a minimal interaction. But since formally $I$ gives rise to the correct $B$-equation of motion it may nevertheless be meaningful to compute its Dirac-anomaly, i.e. its response under a change of Dirac-brane. One has $C^{\prime}=C+d W, B^{\prime}=B-g W$ where (see (2.1) $) W$ is the $\delta$ function on a manifold $T$ whose boundary is made out of the old and new Dirac-branes. $I$ changes then by

$$
\Delta I=\frac{g^{2}}{G} \int W J
$$

where the integral would count the number of intersections between $M$ and $T$. But since $M$ is a submanifold of $T$ this integral is ill-defined.

The situation can be saved by introducing a framing regularization. We replace the surface $M$ in $\int_{M} B$ with a surface $M_{\varepsilon}$ (and the current $J$ with $J_{\varepsilon}$ ) obtained from $M$ by displacing each point of $M$ by an amount $\varepsilon$ in an arbitrary direction. This gives instead of $\Delta I$

$$
\Delta I_{\varepsilon}=\frac{g^{2}}{G} \int W J_{\varepsilon}
$$

where the integral is now well-defined and integer. The Dirac-brane is then unobservable if the charge is quantized as

$$
\frac{g^{2}}{G}=2 \pi n .
$$

One may ask if this simple regularization procedure could be invoked also to regularize the self-interactions (1.5) of a magnetic brane. This is not the case, for two reasons: first, the terms in (1.5) are non-linear (quadratic) while the equation of motion for a chiral boson $(H=* H)$ is linear and, second, the equation of motion itself is well-defined, while the non-linear terms in (1.5) involve $\left.B\right|_{M}$ and are ill-defined.

2) Chern-kernels. We can introduce an odd Chern-kernel such that $J=d K$ and write

$$
H=d B+g K
$$

In this case the potential is regular on $M$, the integral $\int_{M} B$ is well-defined and we have only to worry about $Q$-invariance. As we know, the pullback of $B$ changes according 
to an $S O(2 N+2)$-transformation, $\left.\delta B\right|_{M}=-g \chi^{1}(a)$, and this implies that $I$ carries the normal bundle gravitational anomaly

$$
\delta I=\frac{g^{2}}{G} \int_{M} \chi^{1}(a)
$$

corresponding to the anomaly polynomial $\frac{g^{2}}{G} \chi(f)$, i.e. to the $S O(2 N+2)$-Euler form. A consistent dynamics requires then the cancellation of this classical anomaly, for example by the quantum anomaly produced by chiral fields localized on $M$.

In conclusion, for self-dual branes both possibilities - Dirac-branes and Chern-kernels - are available, and which one has to be used depends on the physical content of the theory. In the absence of quantum gravitational anomalies one would use Dirac-branes together with a framing regularization and impose charge quantization, while in their presence Chern-kernels can play a central role in their cancellation upon choosing particular values for the charges. The availability of this second possibility is indeed crucial for the consistency, at the quantum level, of the situation considered in the next section.

\section{$5 \quad M 2$-branes ending on $M 5$-branes}

The effective action for closed $M 5$-branes interacting with closed $M 2$-branes through eleven-dimensional supergravity has been constructed in [7; it employs a four-form Chern-kernel for the M5-brane current and realizes the cancellation of the residual M5brane normal bundle anomaly, which is an $S O(5)$-anomaly. The $M 2$-brane carries an odd-dimensional worldvolume and entails no anomalies; it is moreover closed, so the coupling to the $M 5$-brane could be performed in a standard way introducing a Dirac-threebrane, as explained in section two of the present paper, and the dynamics is quantum mechanically consistent if charge quantization holds.

But in eleven-dimensional space-time an M2-brane can have also an open worldvolume, under the condition that its boundary belongs to an $M 5$-brane [16]; in this respect M5-branes can be really considered as Dirichlet-branes for eleven-dimensional membranes, as pointed out in [17]. The principal differences w.r.t to closed $M 2$-branes are the following; first, for open membranes no natural Dirac-brane can be defined (and no one is needed); second, the boundary of the membrane is a string describing a two-dimensional worldvolume and as such it is plagued by gravitational anomalies [6]; third, since open membranes must end on 5-branes the interaction between the two surfaces is more intricate then in the closed case where the relative position of the two surfaces is arbitrary; the open membrane exhibits instead a contact-interaction with the 5-brane and this leads a priori to additional singularities located at its boundary. The boundary of the membrane couples, moreover, minimally to the chiral two-form present on the 5-brane and so it becomes actually a self-dual string.

We will show that all these problematic features are naturally and elegantly solved by the Chern-kernel approach, if the $M 2$-brane charge $e$ and the $M 5$-brane charge $g$ assume 
their $M$-theory values

$$
g^{3}=2 \pi G, \quad e=g^{2},
$$

leading for the brane tensions to the standard relations [18, 19]

$$
T_{g}^{3}=\frac{2 \pi}{G^{2}}, \quad T_{e}=G T_{g}^{2}
$$

The results of the present section are summarized in the Wess-Zumino action (5.15), based on an odd and an even Chern-kernel, which cancels the quantum gravitational anomalies of the system.

\subsection{Normal bundles and quantum gravitational anomalies}

Calling the $M 2$-brane worldvolume $M_{3}$, its boundary $M_{2}$ and the $M 5$-brane worldvolume $M_{6}$ we have

$$
\partial M_{3}=M_{2} \subset M_{6}, \quad \partial M_{6}=0=\partial M_{2} .
$$

On the $M 5$-brane the eleven-dimensional Lorentz group $S O(1,10)$ reduces to the structure group

$$
S O(1,5) \times S O(5)
$$

where $S O(1,5)$ is its tangent group and $S O(5)$ the invariance group of its normal bundle. Since there are chiral fields localized at $M_{6}$, i.e. the chiral two-form potential $b$ and the 32-component Green-Schwarz spinor $\vartheta$ of $S O(1,10)$, the structure group is plagued by gravitational anomalies. The associated $M 5$-brane eight-form anomaly polynomial can be rewritten as the sum [20],

$$
2 \pi\left(\left.I_{8}\right|_{M_{6}}+\frac{1}{24} P_{8}\right)
$$

where $P_{8}(f)$ is the second Pontrjagin form of the normal bundle $S O(5)$-curvature $f$, and $I_{8}(R)$ is the polynomial of the target-space $S O(1,10)$-curvature $R$, which corrects elevendimensional supergravity by the term $\int B I_{8}$ [18. Clearly, since $\left.I_{8}\right|_{M_{6}}$ can be written in terms of normal and tangent curvatures, the $S O(1,5)$-tangent bundle is anomalous as well.

On $M_{2} \subset M_{6}$ the structure group (5.3) reduces further according to

$$
\begin{aligned}
S O(1,5) & \rightarrow S O(1,1) \times S O_{a}(4) \\
S O(5) & \rightarrow S O_{b}(4)
\end{aligned}
$$

where $S O_{a}(4)$ is the normal group of $M_{2}$ with respect to $M_{6}$, and $S O(1,1)$ is its tangent group. The reduction of $S O(5)$ to $S O_{b}(4)$ occurs along the component normal to $M_{6}$ of the unique direction on $M_{2}$ which is normal to $M_{2}$ and tangent to $M_{3}$. In conclusion, the structure group on $M_{2}$ is

$$
S O(1,1) \times S O_{a}(4) \times S O_{b}(4)
$$


The Green-Schwarz spinor reduces on $M_{2}$ to a set of fermions which are chiral w.r.t this group and they give therefore rise to gravitational anomalies; the corresponding four-form anomaly polynomial has been determined in [6] and reads ${ }^{9}$

$$
\pi\left(\chi_{b}-\chi_{a}\right)
$$

where $\chi_{a, b}$ are the Euler-forms of the $S O_{a, b}(4)$-bundles. Notice in particular that the $S O(1,1)$-tangent bundle is anomaly free.

A consistent low energy effective action for the system $M 2+M 5$ must therefore cancel simultaneously the anomaly (5.4) supported on $M_{6}$, and the anomaly (5.5) supported on $M_{2}$.

\subsection{Currents, Chern-kernels and equations of motion}

In this section we have to distinguish target-space forms, defined in eleven-dimensional space-time, from forms defined only on $M_{6}$. Generically we will indicate the former with capital letters and the latter with small letters. One has to take some care when considering products of forms which are defined on different manifolds. Indeed, in general the formal product $\phi_{1} \phi_{2}$ between forms defined on different manifolds does not define a form, but it defines a form say on $M_{2}$ if $\phi_{2}$ is the $\delta$-function on $M_{1}$ w.r.t. $M_{2}$ and $\phi_{1}$ is a form on $M_{1}$, see [7, 14].

We indicate the Poincaré-duals of $M_{6}, M_{3}$ and $M_{2}$ respectively with $J_{5}, J_{8}$ and $J_{9}$, where the subscripts indicate the degrees of the forms. Since $M_{2}$ is a submanifold of $M_{6}$ we can also define the Poincaré-dual of $M_{2}$ w.r.t. to $M_{6}$ : this is a four-form on $M_{6}$ which we indicate with $j_{4}$. Then the relations (5.2) translate into

$$
d J_{8}=J_{9}, \quad j_{4} J_{5}=J_{9}, \quad d J_{5}=0=d j_{4} .
$$

The starting point for the construction of the bosonic effective action for $D=11$ supergravity interacting with $M 2$ - and $M 5$-branes is a set of consistent equations of motion for the physical fields of the system. These are the space-time metric and a three-form potential $B$ in the target-space $M_{11}$, the coordinates and a (Born-Infeld like) chiral two-form $b$ on $M_{6}$, and the coordinates on $M_{3}$. Concentrating on the dynamics of the potentials $b$ and $B$ and calling their field-strengths $h$ and $H$ respectively we can write their Bianchi identities (magnetic equations) and equations of motion as

$$
\begin{aligned}
d H & =g J_{5} \\
d * H & =\frac{1}{2} H H+g h J_{5}+g^{2} I_{8}+e J_{8} \\
d h & =\left.d B\right|_{M_{6}}+\frac{e}{g} j_{4} \\
h & =* h+(\text { n.l.t. }),
\end{aligned}
$$

\footnotetext{
${ }^{9}$ With respect to reference [6] we added a factor of $1 / 2$ since the Green-Schwarz fermion is Majorana.
} 
where the non-linear terms in the self-duality equation for $h$ (5.10) correspond to the Born-Infeld lagrangian, see the next section. The form of these equations is almost uniquely fixed by the requirement of generalized charge conservation for $H$, i.e. by the requirement that the r.h.s. of (5.8) is a closed form ${ }^{10}$. However, to check this closure one must first express $H$ and $h$ in terms of $B$ and $b$, solving (5.7) and (5.9). According to the strategy developed in this paper we introduce an even $S O(5)$ four-form Chern-kernel $K$ for $J_{5}$, a target-space form, and an odd $S O_{a}(4)$ three-form Chern-kernel $k$ for $j_{4}{ }^{11}$, a form on $M_{6}$ :

$$
J_{5}=d K, \quad j_{4}=d k .
$$

The $S O(5)$-kernel is written explicitly in (B.1) and the $S O_{a}(4)$-kernel is just the sum of (3.31) and (3.32). The choice of a Chern-kernel for $j_{4}$, instead of a Dirac-brane, is advisable because $M_{2}$ is a self-dual string plagued by quantum gravitational anomalies, [see (5.5)]. On the other hand, the presence of the products $h J_{5}$ and $H H$ in (5.8) forbids the use of Dirac-branes also for $J_{5}$ : the first term contains $B J_{5}$ and $d b J_{5}$, see (1.5), and the second term, using a Dirac-brane $C_{g}$ instead of the Chern-kernel $K$, would contain a term $C_{g} C_{g}$, which is the square of a $\delta$-function.

The field strengths read then

$$
\begin{aligned}
H & =d B+g K \\
h & =d b+\left.B\right|_{M_{6}}+\frac{e}{g} k .
\end{aligned}
$$

With these determinations, using (15.6) and the product formulae for even Chern-kernels of section three, it is straightforward to verify that (5.8) closes.

Since $k$ is odd, under an $S O_{a}(4)$-transformation on $M_{2}$ we have, as remnant of the $Q$-transformation of $b$, the anomalous transformation law

$$
\left.\delta b\right|_{M_{2}}=-\frac{e}{g} \chi_{a}^{1}
$$

\subsection{Anomaly free effective action}

We write the effective action for our system as

$$
\Gamma=\frac{1}{G}\left(S_{k i n}+S_{w z}\right)+\Gamma_{q}
$$

where $\Gamma_{q}$ is the quantum effective action carrying the anomalies (5.4) and (5.5), and the classical action $S_{k i n}+S_{w z}$ must by definition give rise to the equations of motion (5.8) and (5.10). The kinetic terms are the standard ones,

$$
S_{k i n}=\int_{M_{11}} d^{11} x \sqrt{-g} R-\frac{1}{2} \int_{M_{11}} H * H-g \int_{M_{6}} d^{6} \sigma \sqrt{-g} \mathcal{L}(h)-e \int_{M_{3}} d^{3} \sigma \sqrt{-g},
$$

\footnotetext{
${ }^{10}$ Our differential acts from the right: $d\left(\Phi_{m} \Phi_{n}\right)=\Phi_{m} d \Phi_{n}+(-)^{n} d \Phi_{m} \Phi_{n}$.

${ }^{11}$ Remember that $S O_{a}(4)$ is indeed the normal group of $M_{2}$ with respect to $M_{6}$.
} 
where $\mathcal{L}(h)$, made out of the Born-Infeld Lagrangian (written in a manifestly covariant way a la PST [21, see subsection 6.1), contains the kinetic terms for the M5-brane coordinates as well as for $b$. Here it suffices to know that it depends on $b$ only through the invariant field strength $h$.

The Wess-Zumino term is written usually as the integral of an eleven-form; here we choose the equivalent alternative to write it as the integral of a closed twelve-from on a twelve-dimensional manifold with boundary,

$$
S_{w z}=\int_{M_{11}} L_{11}=\int_{M_{12}} L_{12}, \quad L_{12}=d L_{11}, \quad \partial M_{12}=M_{11} .
$$

We are here assuming the absence of topological obstructions. One advantage of this procedure is the manifest invariance of $L_{12}$ under all relevant symmetries, while usually $L_{11}$ is invariant only up to a closed form. This approach requires to extend the targetspace fields to $\mathbf{R}^{12}$, and in particular to extend every involved $p$-brane to a $(p+1)$-brane whose restriction on $M_{11}$ reduces to the $p$-brane [7]. Closed branes extend to closed branes, and the boundary of the extended M2-brane has to belong to the extended M5brane. This procedure maintains then the degrees of the current-forms $J_{5}, J_{8}$ etc. and hence of the Chern-kernels and of the dimensions of the normal bundles. With an abuse of language we indicate the extended worldvolumes still with their eleven-dimensional names, e.g. $M_{6}$, even if it is now a six-brane with a seven-dimensional worldvolume.

The crucial ingredient of this construction is then the twelve-form

$$
\begin{aligned}
L_{12}= & \frac{1}{6} H H H+\frac{g}{2} h d B J_{5}+g^{2} I_{8} H+g^{3}\left(I_{7}^{0}+\frac{1}{24} P_{7}^{0}\right) J_{5} \\
& +e H J_{8}+\frac{e}{2}\left(d b+B+g \chi_{b}^{0}\right) J_{9},
\end{aligned}
$$

where we introduced the canonical Chern-Simons forms: $I_{8}=d I_{7}^{0}, P_{8}=d P_{7}^{0}, \chi_{b}=d \chi_{b}^{0}$. This formula is the unique solution to the following three requirements:

1) It must give rise to the equations of motion (5.8) and (5.10).

2) It must be closed.

3) It must be $Q$-invariant in the bulk, i.e. apart from terms supported on the branes.

The equations of motion fix indeed all terms in $L_{12}$ which depend on $B$ or $b$. The terms which are independent of these fields are fixed by the requirements 2) and 3). The formula is, actually, manifestly invariant under $Q$-transformations of $B$ : this potential shows up in the combination $H$ or as $\left.B\right|_{M_{6}}$ and both are invariant. The potential $b$ shows up as $h$, which is invariant, and as $d b J_{9}$ which transforms by an $S O_{a}(4)$-transformation according to (5.13). Eventually, the terms $g^{3}\left(I_{7}^{0}+\frac{1}{24} P_{7}^{0}\right) J_{5}$ and $\frac{g e}{2} \chi_{b}^{0} J_{9}$ are needed to make $L_{12}$ a closed form, as can be seen using - before differentiation - the product formulae of section three ${ }^{12}$. The unique non trivial point, in checking that $d L_{12}=0$, is to show that there

\footnotetext{
${ }^{12}$ That $L_{12}$ is closed for $e=0$, i.e. in the absence of $M 2$-branes, has been shown in [7].
} 
exists a three-form $X$ on $M_{3}$ such that ${ }^{13}$

$$
K J_{8}+\frac{1}{2} \chi_{b}^{0} J_{9}=d\left(X J_{8}\right)
$$

A proof of this relation, together with an explicit expression for $X$, are reported in appendix $B$. For our purposes the defining relation (5.16) is sufficient, because it determines all properties of $X J_{8}$ modulo a closed form.

Another important point regarding $L_{12}$ is that the Chern-Simons forms $P_{7}^{0}$ and $\chi_{b}^{0}$ appear evaluated respectively on the worldvolumes $M_{6}$ and $M_{2}$, so that no unphysical extensions of the $S O(5)$ - and $S O_{b}(4)$-connections show up.

Eventually we can also give an explicit expression for the Wess-Zumino action, written in eleven-dimensional space $\left(d L_{11}=L_{12}\right)$,

$$
\begin{aligned}
L_{11}= & \frac{1}{6} B d B d B+\frac{g}{2} B d B K-\frac{g}{2} b\left(d B+\frac{e}{g} j_{4}\right) J_{5}+g^{2} B\left(I_{8}+\frac{1}{2} K K\right) \\
& +e B\left(J_{8}+\frac{1}{2} k J_{5}\right)+g^{3}\left(I_{7}^{0}+\frac{1}{24}\left(P_{7}^{0}+Y_{7}\right)\right) K+e g X J_{8},
\end{aligned}
$$

where the invariant form $Y_{7}$ is defined in (3.22) - see [7] for an explicit expression - and we recall that $K K=\frac{1}{4} d\left(P_{7}^{0}+Y_{7}\right)$. The $b$-dependence of $L_{11}$ is fixed by the Bianchi identity (5.9) and by the PST-symmetries or, equivalently, by the equation of motion (5.10). To derive the $B$-equation of motion (5.8) from $\frac{1}{G}\left(S_{k i n}+\int_{M_{11}} L_{11}\right)$, the only non trivial point is the knowledge of the variation of the Born-Infeld action under a generic variation of $B$; taking advantage from (5.10) one obtains [21],

$$
\delta \int_{M_{6}} d^{6} \sigma \sqrt{-g} \mathcal{L}(h)=\frac{1}{2} \int_{M_{6}} h \delta B=-\frac{1}{2} \int_{M_{11}} h J_{5} \delta B .
$$

The integral $\int L_{11}$ is clearly also invariant under the ordinary gauge transformations

$$
\delta B=d \Lambda, \quad \delta b=d \lambda-\left.\Lambda\right|_{M_{6}}
$$

\section{$5.4 Q$-invariance and gravitational anomalies}

The terms in $L_{11}$ which are not fixed by the equations of motion are the ones multiplying $g^{3}$ and $e g$ in the second line of (5.17); they are indeed crucial to establish $Q$-invariance of the Wess-Zumino action and they introduce, in turn, gravitational anomalies.

The invariance under $Q$-transformations for $B$ of the Wess-Zumino action in its eleven-dimensional form is less obvious than in its manifestly invariant twelve-dimensional form; for $e=0$ this invariance has been explicitly verified in [7, proving the necessity of the $g^{3}$-terms. The necessity of the term $\operatorname{eg} X J_{8}$ is again a consequence of this invariance; under

$$
K \rightarrow K+d Q, \quad B \rightarrow B-g Q
$$

\footnotetext{
${ }^{13}$ This relation requires the consistency condition $d\left(K J_{8}\right)=\frac{1}{2} \chi_{b} J_{9}$, which is indeed true, but to prove it one can not use naively Leibnitz's rule, see appendix $B$.
} 
concentrating on the terms proportional to $e$ in $L_{11}$, one is left with the $Q$-variation

$$
\Delta\left(e B J_{8}\right)=-e g Q J_{8}
$$

which should cancel against the variation of $\operatorname{eg} X J_{8}$. The transformation property of $X$ can be deduced from its defining relation (5.16),

$$
d\left(\Delta X J_{8}\right)=d Q J_{8}=d\left(Q J_{8}\right)-Q J_{9}=d\left(Q J_{8}\right)
$$

where $Q J_{9}$ vanishes because $Q$ vanishes on $M_{6}$, and hence also on $M_{2}$. This gives

$$
\Delta \int_{M_{11}} X J_{8}=\int_{M_{11}} Q J_{8}
$$

and

$$
\Delta \int_{M_{11}}\left(e B J_{8}+e g X J_{8}\right)=0
$$

This proves that $X J_{8}$ is necessary to restore $Q$-invariance of $S_{w z}$. On the other hand, (5.16) implies also that under $S O_{b}(4)$-transformations one has

$$
d\left(\delta X J_{8}\right)=-\frac{1}{2} d\left(\chi_{b}^{1} J_{9}\right)
$$

and therefore

$$
\delta \int_{M_{11}}\left(e g X J_{8}\right)=-\frac{e g}{2} \int_{M_{2}} \chi_{b}^{1}
$$

which represents a gravitational anomaly.

Under $Q$-transformations of $b$ (see (5.13) $)$

$$
k \rightarrow k+d q, \quad b \rightarrow b-\frac{e}{g} q,\left.\quad q\right|_{M_{2}}=\chi_{a}^{1}
$$

the Wess-Zumino action is invariant up to a gravitational anomaly, as can be seen by direct inspection,

$$
\delta \int_{M_{11}} L_{11}=\frac{e^{2}}{2 g} \int_{M_{2}} \chi_{a}^{1}
$$

Eventually one can compute the gravitational anomalies carried by the Wess-Zumino action. Using (5.15) and (5.13) (or (5.17), (5.19) and (5.20) ) one sees that there are anomalies supported on $M_{6}$ and $M_{2}$,

$$
\delta\left(\frac{1}{G} S_{w z}\right)=-\frac{g^{3}}{G} \int_{M_{6}}\left(I_{6}^{1}+\frac{1}{24} P_{6}^{1}\right)-\frac{e g}{2 G} \int_{M_{2}}\left(\chi_{b}^{1}-\frac{e}{g^{2}} \chi_{a}^{1}\right) .
$$

These classical anomalies cancel just against the quantum ones, (5.4) and (5.5), if the charges satisfy (5.1).

We may rephrase this result as follows. Once we have introduced the Chern-kernels $K$ and $k$, it is straightforward to write a "minimal" classical action which gives rise to the equations of motion (5.8) and (5.10), since all terms depending on $B$ and $b$ are fixed. 
This action is $\frac{1}{G}\left(S_{k i n}+\int \widetilde{L}_{11}\right)$, where $\widetilde{L}_{11}$ is given in (5.17) but without the terms in $g^{3}$ and $e g$. This would lead to the total effective action (classical + quantum)

$$
\widetilde{\Gamma}=\frac{1}{G}\left(S_{k i n}+\int \widetilde{L}_{11}\right)+\Gamma_{q}
$$

Since $\frac{1}{G} \int \widetilde{L}_{11}$ carries already the $S O_{a}(4)$-anomaly polynomial $\pi \chi_{a}$ on $M_{2}$, the effective action $\widetilde{\Gamma}$ is plagued by the gravitational anomalies $2 \pi\left(I_{8}+\frac{1}{24} P_{8}\right)$ on $M_{6}$ and $\pi \chi_{b}$ on $M_{2}$, but also by $Q$-anomalies. What we have shown in this paper is the non-trivial fact that there exists the counterterm

$$
2 \pi \int_{M_{11}}\left(I_{7}^{0}+\frac{1}{24}\left(P_{7}^{0}+Y_{7}\right)\right) K+2 \pi \int_{M_{3}} X
$$

which cancels the gravitational and $Q$-anomalies simultaneously.

The values (5.1) for the charges, which ensure anomaly cancellation, satisfy in particular the Dirac-quantization condition $e g=2 \pi n G$ with $n=1$; this is, actually, a remarkable coincidence since the Dirac-quantization results in general from an unobservabilityrequirement of the Dirac-brane, but in the present case no Dirac-brane at all was introduced, because the M2-brane has a boundary. The physical meaning of this coincidence can be traced back to the limiting case in which the open $M 2$-brane becomes a closed one and leaves the $M 5$-brane. Since the $M 2$-charge remains unchanged during this process and since a closed $M 2$-brane is consistent if its charge satisfies the Dirac-quantization condition, one expects that our Wess-Zumino action for an open membrane reduces to the one of a closed one, if the boundary of the membrane shrinks to zero. In particular, in this limit a Dirac-brane for $M 2$ should in turn appear. This happens, indeed, as follows. As the $M 2$-brane closes one has to take simultaneously the limits

$$
j_{4} \rightarrow 0, \quad J_{9}=d J_{8} \rightarrow 0, \quad k \rightarrow 0
$$

The field strength $h$ reduces to $d b+\left.B\right|_{M_{6}}$, and the unique contribution of order $e$ surviving in (5.15) is $e H J_{8}$. It contributes to the classical action with

$$
S_{e}=\frac{e}{G} \int_{M_{12}} H J_{8}
$$

Since the membrane is now closed, $d J_{8}=0$, we can introduce a Dirac-brane and write $J_{8}=d C_{e}$. Using $e g=2 \pi G$ the interaction above can then be rewritten as

$$
S_{e}=\frac{e}{G} \int_{M_{11}} H C_{e}+2 \pi \int_{M_{12}} J_{5} C_{e}
$$

The second term is an (irrelevant) integer multiple of $2 \pi$ and the first term reproduces the standard interaction between closed branes and dual branes, see (2.7). The integral $\int L_{11}$ reduces correspondingly to the Wess-Zumino action for interacting closed $M 2-$ and M5-branes, constructed in [7]. 
We end this section with a comparison of our approach with the one of [2, adapted according to [4, 5], in absence of M2. Specifically we may compare our Wess-Zumino term (5.17) - with $e=0$ and disregarding the obvious terms containing $I_{8}$ and $I_{7}^{0}$ - with the Chern-Simons term $S_{S C}$, eq. (19) of ref. [4]. In that approach the integration is performed over $M_{11}$ minus a tubular neighborhood of $M_{6}$ and, restricted to this space, $K=\frac{1}{2} e_{4}$ is a closed form, hence locally $e_{4}=d e_{3}$. In this framework, by construction, $S_{C S}$ looses terms supported on $M_{6}$ and one can see that $L_{11}$ differs from $S_{C S}$ indeed by terms proportional to $J_{5}$. These terms are actually needed to get the correct equation of motion (5.8) for $B$.

There remains, however, a difference between $S_{C S}$ and $L_{11}$ also in the bulk, regarding the anomaly cancelling term; this is written in [4] as $\frac{1}{8} e_{3} e_{4} e_{4}$, and in $L_{11}$ as $\frac{1}{4} K_{4}\left(P_{7}^{0}+Y_{7}\right)$. These two eleven-forms can be mapped into one another through a formal integration by parts, using $K_{4}=\frac{1}{2} d e_{3}$. However, due to the presence of the tubular neighborhood this operation leads to additional terms in the action, and it is not clear whether the two cancellation mechanisms are equivalent or not. In our opinion this point requires further investigation.

\section{$6 \quad B_{3} \leftrightarrow B_{6}$ duality-invariant action}

In this section we present a classical action, equivalent to the one constructed in the previous section, which involves the three-form potential $B$ - now we call it $B_{3}$ and the field strength $H_{4}=d B_{3}+g K-$ and its dual six-from potential $B_{6}$, in a duality symmetric and manifestly Lorentz-invariant way. For the problems regarding such a construction when one uses Dirac-branes instead of Chern-kernels see reference [22]. The knowledge of a consistent duality-invariant action may be useful for various purposes, for example for the investigation of the flux quantization conditions of the dual curvature $H_{7}$, or for an analysis of dimensional reductions involving dual branes and dual potentials. Eventually such an action allows also a comparison with the $M 5$-brane $\sigma$-model action [23, 24] (where the supergravity fields are treated as source-less i.e. they satisfy the equations of motion of pure supergravity), because also the construction of the $\sigma$-model action involves necessarily $B_{3}$ as well as $B_{6}$.

\subsection{PST-action for $b$}

We rewrite the classical action of the previous section (disregarding the kinetic terms for the metric and for the $M 2$-brane, which are irrelevant for the present purposes)

$$
S_{0}\left[B_{3}, b, c\right]=-\frac{1}{2} \int_{M_{11}} H_{4} * H_{4}-g \int_{M_{6}} d^{6} \sigma \sqrt{-g} \mathcal{L}(h)+\int_{M_{11}} L_{11} .
$$

In this subsection we review briefly the basic ingredients of the PST-approach for the

self-interacting chiral two-form $b$ [21], specifying in particular the lagrangian $\mathcal{L}(h)$. One 
introduces an auxiliary scalar field $c(\sigma)$ on $M_{6}$, and constructs the unit vector

$$
v_{i}=\frac{\partial_{i} c}{\sqrt{\partial c \partial c}}, \quad v^{i} v_{i}=1 .
$$

Then one can define two two-forms on $M_{6}$, contracting $h$ and $* h$ with this vector:

$$
\mathcal{H} \equiv i_{v} h, \quad \widetilde{\mathcal{H}} \equiv i_{v} * h, \quad h=-(v \mathcal{H}+* v \widetilde{\mathcal{H}})
$$

where $v$ is the one-form $d c / \sqrt{\partial c \partial c}$. In terms of these data one has

$$
\mathcal{L}(h)=\mathcal{L}_{B I}(\widetilde{\mathcal{H}})-\frac{1}{4} \mathcal{H}^{i j} \widetilde{\mathcal{H}}_{i j}, \quad \mathcal{L}_{B I}(\widetilde{\mathcal{H}})=\sqrt{\operatorname{det}\left(\delta_{i}{ }^{j}+i \widetilde{\mathcal{H}}_{i}^{j}\right)}
$$

and the precise form of the equation of motion (5.10) is

$$
\mathcal{H}=\mathcal{W}(\widetilde{\mathcal{H}}) \equiv d \sigma^{i} d \sigma^{j} \frac{\delta \mathcal{L}_{B I}}{\delta \widetilde{\mathcal{H}}^{i j}}
$$

Since we have $\mathcal{W}(\widetilde{\mathcal{H}})=\widetilde{\mathcal{H}}+o\left(\widetilde{\mathcal{H}^{3}}\right)$, and $\mathcal{H}=\widetilde{\mathcal{H}}$ is the same as $h=* h,(6.2)$ is indeed a non-linear version of $h=* h$. After fixing the PST-symmetries, see below, the action $S_{0}$ gives rise to (6.2). This equation of motion can indeed be rewritten in a manifestly Lorentz-invariant way, and the PST-symmetries ensure correspondingly that $c$ is non propagating.

\subsection{The potential $B_{6}$ and its curvature $H_{7}$}

The first step in writing a duality-invariant action consists in the introduction of a dual potential, i.e. in the solution of the $B_{3}$-equation of motion (5.8) in terms of a potential. Once this is done in a consistent way, the construction of the duality-invariant action a la PST is almost canonical.

A consistent reconstruction of a potential $B_{6}$ relies on the fact that the r.h.s. of (5.8) is a well defined invariant closed form, so it can be written as the differential of some seven-form. To make this seven-form explicit we must introduce a new target-space form $\mathrm{O}_{7}$ whose existence is guaranteed by

$$
d\left(k J_{5}+J_{8}\right)=-j_{4} J_{5}+J_{9}=0 \rightarrow k J_{5}+J_{8}=d O_{7} .
$$

This allows to recast the Bianchi identity (5.7) for $B_{3}$ and its equation of motion (5.8), in the equivalent system of equations

$$
\begin{aligned}
& H_{4}=d B_{3}+g K, \\
& H_{7}=d B_{6}+\frac{1}{2} B_{3} d B_{3}+g\left(\widehat{d b}+B_{3}\right) K+g^{2}\left(I_{7}^{0}+\frac{1}{8}\left(P_{7}^{0}+Y_{7}\right)\right)+e O_{7}, \\
& H_{4}=* H_{7} .
\end{aligned}
$$

The dual potential is defined a priori up to a field redefinition; the choice (6.4) corresponds to a choice for $B_{6}$ which is regular on $M_{6}$, as we will see now. The necessity of a regular $B_{6}$ 
results from the fact that the duality-invariant action will contain the minimal coupling $\int B_{6} J_{5}=\int_{M_{6}} B_{6}$.

In the formula for $H_{7}$ we introduced a field $\widehat{b}$ which represents an unphysical (holographic) target-space extension of the two-form potential $b$ on $M_{6}$,

$$
\left.\widehat{b}\right|_{M_{6}}=b,
$$

so let us first establish that the extension is unobservable. Changing the extension we have

$$
\widehat{b}^{\prime}=\widehat{b}+\Delta \widehat{b},\left.\quad \Delta \widehat{b}\right|_{M_{6}}=0
$$

which leads in $H_{7}$ to a change $g d(\Delta \widehat{b}) K=g d(\Delta \widehat{b} K) . H_{7}$ is kept invariant if $B_{6}$ changes accordingly by

$$
B_{6}^{\prime}-B_{6}=-g \Delta \widehat{b} K
$$

Notice that this transformation keeps $\left.B_{6}\right|_{M_{6}}$ invariant $^{14}$.

The field-strength $H_{7}$ contains necessarily singularities near $M_{6}$ and, as in the case of the four-form field-strength $H_{4}$, these singularities have to be universal ${ }^{15}$. To make them explicit we should know the singular behaviour of $O_{7}$ near the $M 5$-brane. From its defining relation - which determines it modulo a closed form - it is clear that there exists a choice, such that its singular part near $M_{6}$ is $k K$. Since $B_{6}, B_{3}, I_{7}^{0}$ and $P_{7}^{0}$ are regular near $M_{6}$, the universal singular behaviours of the curvatures are given by

$$
\begin{aligned}
& {\left[H_{4}\right]_{\text {sing }}=g K,} \\
& {\left[H_{7}\right]_{\text {sing }}=g h K+\frac{g^{2}}{8} Y_{7} .}
\end{aligned}
$$

The consistency of this construction requires now that under $Q$-transformations of $B_{3}, H_{7}$ is invariant in compatibility with a regular transformation law for $B_{6}$ on $M_{6}{ }^{16}$. Due to the presence of the $S O(5)-$ Chern-Simons form $P_{7}^{0}$ in $H_{7}$, one expects that under $Q$-transformations one has the remnant anomalous $S O(5)$-transformation

$$
\left.\Delta B_{6}\right|_{M_{6}}=-\left.\frac{g^{2}}{8} P_{6}^{1}\right|_{M_{6}}
$$

\footnotetext{
${ }^{14}$ There exists an alternative definition of a six-form potential which avoids the extension of $b$ : you can choose the singular potential $\widetilde{B}_{6}=B_{6}+g \widehat{b} K$. This gives$$
H_{7}=d \widetilde{B}_{6}+\frac{1}{2} B_{3} d B_{3}-g b J_{5}+g B_{3} K+g^{2}\left(I_{7}^{0}+\frac{1}{8}\left(P_{7}^{0}+Y_{7}\right)\right)+e O_{7},
$$

and no unphysical extension of $b$ shows up. But with this potential the formula for $H_{7}$ shows up a $\delta$-like singularity on $M_{6}$ (the term $b J_{5}$ ), which is canceled by the singularity contained in $\widetilde{B}_{6}$. Use of the potential $B_{6}$ and of an extended $\widehat{b}$ makes this cancellation explicit.

${ }^{15}$ The pullback of $H_{7}$ to $M_{6}$ vanishes for dimensional reasons, so the singularities we are speaking about are considered in the algebraic sense.

${ }^{16}$ Since the r.h.s. of (5.8) is $Q$-invariant, it is obvious that there exists always a transformation $\Delta B_{6}$ which keeps $H_{7}$ invariant; it is less trivial that $\Delta B_{6}$ is finite on $M_{6}$.
} 
Since $O_{7}$ is $Q$-invariant, to deduce the transformation of $B_{6}$ one must first determine the transformation law of

$$
S \equiv P_{7}^{0}+Y_{7}
$$

Under $K^{\prime}=K+d Q$, since $K K=\frac{1}{4} d S$, one obtains easily

$$
S^{\prime}=S+8 K Q+4 Q d Q+d \mathcal{P}
$$

for some six-form $\mathcal{P}$. But we have also $S^{\prime}=P_{7}^{0 \prime}+Y_{7}^{\prime}$, and since $Y_{7}^{\prime}$ and $Y_{7}$ have the same (singular) behaviour near $M_{6}$ we get $\left.\left(Y_{7}^{\prime}-Y_{7}\right)\right|_{M_{6}}=0$, and therefore

$$
\left.\left(S^{\prime}-S\right)\right|_{M_{6}}=\left.\left(P_{7}^{0 \prime}-P_{7}^{0}\right)\right|_{M_{6}}=\left.d P_{6}^{1}\right|_{M_{6}}
$$

Comparing this with (6.8) and remembering that $\left.Q\right|_{M_{6}}=0$ we get, apart from a closed form,

$$
\left.\mathcal{P}\right|_{M_{6}}=\left.P_{6}^{1}\right|_{M_{6}} .
$$

Using (6.8) it is eventually straightforward to show that $H_{7}$ is $Q$-invariant under

$$
\begin{aligned}
& K^{\prime}=K+d Q \\
& B_{3}^{\prime}=B_{3}-g Q \\
& B_{6}^{\prime}=B_{6}-g\left(d \widehat{b}+\frac{1}{2} B_{3}\right) Q-\frac{g^{2}}{8} \mathcal{P},
\end{aligned}
$$

which proves also that $B_{6}$ has a regular transformation law on $M_{6}$, given by (6.7). Under $S O(1,10)$-transformations one must obviously also have

$$
\delta B_{6}=-g^{2} I_{6}^{1}
$$

$H_{7}$ is also invariant under ordinary gauge transformations,

$$
\delta B_{3}=d \Lambda_{2}, \quad \delta B_{6}=d \Lambda_{5}-\frac{1}{2} \Lambda_{2} d B_{3}, \quad \delta \widehat{b}=d \Lambda_{1}-\Lambda_{2}
$$

\subsection{Duality-invariant action and PST-symmetries}

Once we have introduced a dual potential the construction of a duality-invariant action, according to the PST-approach, requires the introduction of an auxiliary target-space scalar field $C(x)$. Due to the presence of a chiral field on the $M 5$-brane, whose action required already an auxiliary field $c(\sigma)$ on $M_{6}$, as shown in [22] the consistency of the whole construction demands now that $c$ is the pullback of $C$,

$$
c=\left.C\right|_{M_{6}} \text {. }
$$

Apart from this, the construction proceeds as follows. Define the target-space vector

$$
V_{\mu}=\frac{\partial_{\mu} C}{\sqrt{\partial C \partial C}}, \quad V^{\mu} V_{\mu}=1
$$


and the target-space three- and six-forms

$$
f_{3} \equiv i_{V}\left(H_{4}-* H_{7}\right), \quad f_{6} \equiv i_{V}\left(H_{7}-* H_{4}\right),
$$

which realize the decomposition

$$
H_{4}-* H_{7}=-\left(V f_{3}+* V f_{6}\right), \quad V=d C / \sqrt{\partial C \partial C}
$$

Then the duality-invariant action is given in terms of (6.1) by

$$
S\left[B_{3}, B_{6}, \widehat{b}, C\right]=S_{0}\left[B_{3}, b, c\right]+\frac{1}{2} \int_{M_{11}} f_{3} * f_{3} .
$$

In this new framework $H_{4}$ and $H_{7}$ are defined in terms of $B_{3}$ and $B_{6}$ as in (6.3) and (6.4), and the duality relation $H_{4}=* H_{7}$ is promoted to an equation of motion which should be produced by $S$ together with the $b$-equation (6.2) ${ }^{17}$.

We show now that $S$ entails the same dynamics as $S_{0}$, i.e. that the two actions are physically equivalent. The starting point are the equations of motion for $B_{3}, B_{6}, b$ and $C$; the complete set, and their derivation, is given in appendix C. For our purposes it is sufficient to know the equations for $B_{6}$ and $B_{3}$, which read

$$
\begin{aligned}
& d\left(V f_{3}\right)=0 \\
& d\left(V f_{6}\right)=g v(\mathcal{H}-\mathcal{W}) J_{5}-V f_{3} H_{4}
\end{aligned}
$$

where $\mathcal{H}$ and $\mathcal{W}$ are defined in subsection 6.1. Knowledge of these equations is sufficient because the $b$-equation is implied by the $B_{3}$-equation (as a direct consequence of the gauge invariance $\left.\delta B_{3}=d \Lambda_{2}, \delta b=-\left.\Lambda_{2}\right|_{M_{6}}\right)$, and the the $C$-equation is identically satisfied if the other three equations of motion hold. This is a consequence of the fact that $C$ is a non-propagating auxiliary field. The action $S$ is in fact invariant (see appendix $\mathrm{C}$ for the proof) under the PST-transformations $(I)$

$$
\widehat{\delta b}=d C \Psi_{1}, \quad \delta B_{3}=d C \Psi_{2}, \quad \delta B_{6}=d C \Psi_{5}, \quad \delta C=0,
$$

with transformation parameters $\Psi_{1}, \Psi_{2}$ and $\Psi_{5}$, and under $(I I)$

$$
\begin{aligned}
\delta C & =\Phi, \\
\delta \widehat{b} & =\Phi\left[\frac{1}{\sqrt{\partial c \partial c}}(\mathcal{H}-\mathcal{W})\right], \\
\delta B_{3} & =\frac{\Phi}{\sqrt{\partial C \partial C}} f_{3}, \\
\delta B_{6} & =\frac{\Phi}{\sqrt{\partial C \partial C}} f_{6}-\frac{1}{2} B_{3} \delta B_{3}-g \delta \widehat{b} K,
\end{aligned}
$$

\footnotetext{
${ }^{17}$ Despite the explicit appearance of $\widehat{b}$ in $H_{7}$ the action $S$ is actually only a functional of $b$, as explained in the previous subsection.
} 
where $\Phi$ is a scalar transformation parameter; with $\left[\frac{1}{\sqrt{\partial c \partial c}}(\mathcal{H}-\mathcal{W})\right]$ we indicate an arbitrary target-space extension of the two-form between brackets.

$\Phi$ being arbitrary, the symmetry $(I I)$ ensures that the auxiliary field $C$ is non propagating, and the symmetries $(I)$ allow to reduce the equations of motion (6.17) and (6.18) to

$$
H_{4}=* H_{7}, \quad \mathcal{H}-\mathcal{W}=0
$$

which proves that $S$ and $S_{0}$ are equivalent. To see how this happens consider the most general solution of (6.17),

$$
V f_{3}=d C d \widetilde{\Psi}_{2}
$$

for some two-form $\widetilde{\Psi}_{2}$. Since under a transformation $(I)$ of $B_{3}$ we have $V f_{3} \rightarrow V f_{3}-$ $d C d \Psi_{2}$, we can use this symmetry (with $\Psi_{2}=\widetilde{\Psi}_{2}$ ) to set $V f_{3}=0$. This is the same as

$$
f_{3}=0
$$

because $i_{V} f_{3}=0$ identically. At this point, taking the differential of (6.18) one gets ${ }^{18}$

$$
d(v(\mathcal{H}-\mathcal{W}))=0
$$

and one can use the same procedure as above - using the symmetry $(I)$ of $\widehat{b}$ - to reduce this equation to

$$
\mathcal{H}-\mathcal{W}=0 .
$$

This simplifies eventually the $B_{3}$-equation to $d\left(V f_{6}\right)=0$, and the symmetry $(I)$ of $B_{6}$ can be used to reduce it further to $f_{6}=0$. The identity (6.15) completes then the proof.

\subsection{Comparison with the $M 5$-brane $\sigma$-model}

The action presented in the present paper describes a fully interacting theory of dynamical supergravity with dynamical brane-like sources, as is appropriate to discuss, for example, anomaly cancellation. On the contrary the $M 5$-brane $\sigma$-model action [23, 24] constrains the supergravity fields to satisfy the (source-less) equations of motion of pure $D=11$ Sugra, as required by $\kappa$-symmetry. In the comparison this fundamental difference has to be taken into account.

The bosonic part of the $\sigma$-model action reads

$$
S^{\sigma}=-g \int_{M_{6}} d^{6} \sigma \sqrt{-g} \mathcal{L}(h)+g \int_{M_{6}}\left(B_{6}-\frac{1}{2} b d B_{3}\right),
$$

where $h=d b+\left.B_{3}\right|_{M_{6}}$, since we disregard here the $M 2$-brane $(e=0)$. The structure of the Wess-Zumino term (the second integral) is fixed by invariance under the ordinary gauge transformations (6.14). Since the $\sigma$-model action contains as main building block the

\footnotetext{
${ }^{18}$ The $b$-equation of motion is precisely obtained by taking the differential of the $B_{3}$-equation, see appendix C.
} 
standard minimal coupling term $\int_{M_{6}} B_{6}=\int_{M_{11}} J_{5} B_{6}$, to make the comparison we should view our action $S$ from the $B_{6}$-point-of-view. This can be done using the identity

$$
-\frac{1}{2}\left(H_{4} * H_{4}-f_{3} * f_{3}\right)=\frac{1}{2}\left(H_{7} * H_{7}+f_{6} * f_{6}\right)-H_{4} H_{7},
$$

which is once more a consequence of (6.15). This allows to rewrite $S$ as

$$
\begin{aligned}
S & =\widehat{S}_{k i n}+\widehat{S}_{w z} \\
\widehat{S}_{k i n} & =\frac{1}{2} \int_{M_{11}}\left(H_{7} * H_{7}+f_{6} * f_{6}\right)-g \int_{M_{6}} d^{6} \sigma \sqrt{-g} \mathcal{L}(h), \\
\widehat{S}_{w z} & =\int_{M_{11}}\left(L_{11}-H_{4} H_{7}\right) .
\end{aligned}
$$

This form of the action privileges indeed the role of the potential $B_{6}$ : it appears only through the canonical kinetic term $\frac{1}{2} H_{7} * H_{7}$ (apart from the square of the six-form $f_{6}$ which vanishes on-shell), and through the standard minimal coupling to the $M 5$-brane contained in $H_{4} H_{7}$. The kinetic term for the $M 5$-brane in $\widehat{S}_{k i n}$ is already the same as in $S^{\sigma}$, and the Wess-Zumino action $\widehat{S}_{w z}$ can be split in the three contributions,

$$
\begin{aligned}
\widehat{S}_{w z} & =S_{w z}^{\text {sugra }}+S_{w z}^{\sigma}+S_{w z}^{\text {int }} \\
S_{w z}^{\text {sugra }} & =-\frac{1}{3} \int_{M_{11}} B_{3} d B_{3} d B_{3}, \\
S_{w z}^{\sigma} & =g \int_{M_{6}}\left(B_{6}-\frac{1}{2} b d B_{3}\right) \\
S_{w z}^{\text {int }} & =-g \int_{M_{11}}\left(B_{3} d B_{3} K+g B_{3} K K+\frac{g^{2}}{12}\left(P_{7}^{0}+Y_{7}\right) K\right)+g \int_{M_{6}} b d B_{3},
\end{aligned}
$$

where all three contributions are separately invariant under the ordinary gauge transformations (6.14). $S_{w z}^{\text {sugra }}$ represents the pure supergravity contribution: the coefficient $-1 / 3$ instead of $+1 / 6$ of the standard $B_{3}$-based Sugra is due to the fact that this scheme privileges $B_{6}$. $S_{w z}^{\sigma}$ is indeed the $\sigma$-model Wess-Zumino action showing up in $S^{\sigma}$. Very naively one could have expected that the total Wess-Zumino action is simply $S_{w z}^{s u g r a}+S_{w z}^{\sigma}$, but the fact is that while $S_{w z}^{\sigma}$ is $Q$-invariant (modulo gravitational anomalies) $S_{w z}^{\text {sugra }}$ is not. The additional interaction terms of $S_{w z}^{\text {int }}$ (of order $g, g^{2}$, and $g^{3}$ ) cure this non-invariance. By direct inspection one sees indeed that

$$
S_{w z}^{s u g r a}+S_{w z}^{i n t}=-2 \int_{M_{11}}\left(L_{11}-g^{2} H_{4} I_{7}^{0}\right)
$$

which is manifestly $Q$-invariant (modulo gravitational anomalies). Eventually, it is only the total action, Wess-Zumino + kinetic terms, that is invariant under PST-symmetries.

Finally it is instructive to analyze how the duality-invariant action realizes the cancellation of the gravitational $M 5$-brane anomalies. $S_{w z}^{s u g r a}$ is invariant and $S_{w z}^{\sigma}$ carries the anomaly (see (6.7) and (6.13) $)$,

$$
-\left(I_{8}+\frac{1}{8} P_{8}\right)
$$


but this would not be the right amount to cancel the quantum anomaly $I_{8}+\frac{1}{24} P_{8}$. But also $S_{w z}^{\text {int }}$ carries an anomaly, $\frac{1}{12} P_{8}$, and it ensures hence the matching; gravitational anomalies and $Q$-invariance are once more entangled.

Our duality invariant-action $S$ can be compared also with the duality-invariant action $S_{B B S}$ of [22, formula (5.28), that is based on a Dirac-brane approach. In that action the (ill-defined) products of $\delta$-functions on Dirac-branes - like $C_{g} C_{g}$ - are ignored. This implies that $S_{B B S}$ contains at most terms linear in $g$ and that it carries no gravitational anomalies. Correspondingly one can see that our action (6.16) (for $e=0$ ) reduces to $S_{B B S}$ - after correcting a factor $1 / 2$ - if one neglects all terms of order $g^{2}$ and $g^{3}$, and replaces the Chern-kernel $K$ with the $\delta$-function on the Dirac-brane $C_{g}$.

\section{Concluding remarks and open problems}

There are attempts in the literature to construct low energy effective actions for $M 5$ branes (with or without $M 2$-branes) relying on potentials $B_{3}$ which are ill-defined on the M5-brane worldvolume [22, 25, 26, 27]; they are based on Dirac-branes, or something equivalent to them, and a consistent treatment of the resulting singularities is still missing 19. These approaches refrain, in particular, from specifying the singular behaviour of the field strength $H_{4}$ near the $M 5$-brane and they entail correspondingly two main drawbacks: the dynamics i.e. the action - but even the equations of motion - contain ill-defined objects, such as the pullback of $B_{3}$ or - even worse - of $H_{4}$ and, moreover, the (formal) actions which result from these approaches do not cancel the gravitational anomalies. The Chern-kernel approach solves these two problems simultaneously.

In this paper we considered a topologically trivial space-time. Nevertheless the WessZumino action

$$
S_{w z}=\int_{M_{12}} L_{12}
$$

with $L_{12}$ given in (5.15), should make sense also in a target-space $M_{11}$ with non-trivial topological properties. In such a space-time the crucial feature is the dependence of $S_{w z}$ on the chosen twelve-manifold $M_{12}$ with boundary $M_{11}$. In the absence of global quantum anomalies one would require that $\frac{1}{G} S_{w z}$ is independent of the chosen $M_{12}$ modulo $2 \pi$. This would be equivalent to require that

$$
\frac{1}{G} \int_{M} L_{12}=2 \pi n,
$$

with $n$ integer, for a generic closed manifold $M$. However, as shown in [28, 29], even in the absence of $M 2$ - and $M 5$-branes the above integral is in general only an integer multiple of $\pi$, meaning that $\exp \left(\frac{i}{G} S_{w z}\right)$ carries a residual sign-dependence on $M_{12}$. But in [28] it is then also shown that the quantum effective action carries a global anomaly,

\footnotetext{
${ }^{19}$ Since in the $M 5$-brane effective action the interaction is cubic and since products of $\delta$-functions do not define distributions the authors think that for this case a consistent treatment can not be achieved.
} 
due to the fermionic determinant of the Rarita-Schwinger operator, which compensates precisely this sign-ambiguity.

A similar situation is supposed to arise in the presence of $M 2-$ and $M 5$-branes. In this case there are additional global quantum anomalies [20, 29] due to the branes, but one expects again that the total effective action is well-defined. This conjecture needs still to be proved, but since the formula (5.15) is explicit and involves only objects with a simple and clear geometrical meaning, we hope to be able to provide a proof based on that formula.

In the absence of branes the proof of cancellation of global anomalies of reference [28] relied on the statement that $H$ defines a half-integral cohomology class, i.e. $[H] \in \frac{g}{2} \mathbf{Z}$. In presence of an $M 5$-brane a necessary condition for global anomaly cancellation is then that $H$ represents a half-integral class in the complement of $M 5$. We can check here that this minimal condition can be easily satisfied in our construction. The first step concerns the definition of $H$ in terms of the Chern-kernel $K$. In a topologically non-trivial spacetime the form $K$ must have the following defining properties: 1) near $M 5$ it behaves as in (3.14) and 2) in the complement of M5 it amounts to a closed form. An explicit realization like (3.14) is still available if there exists a map $y^{a}(x)$ from target-space to $\mathbf{R}^{5}$, reducing to normal coordinates in a tubular neighborhood of $M 5$ and nowhere vanishing in its complement. Defining $\hat{y}^{a}=y^{a} /|y|$ we can then introduce a globally defined four-form $K$ according to (3.14) and write

$$
H=H_{0}+g K
$$

where $H_{0}$ (locally $d B_{3}$ ) is a half-integral cohomology class, $\left[H_{0}\right] \in \frac{g}{2} \mathbf{Z}$, as assumed in 28 ] in absence of branes. Using the above realization for $K$ we can now show that also

$$
[K] \in \mathbf{Z} / 2 \text { in the complement of } M 5 \text {. }
$$

Take in each point of target-space an $S O(4)$-basis $n^{a r}(x)$ normal to $\hat{y}^{a}, n^{a r} \hat{y}^{a}=0$, $n^{a r} n^{a s}=\delta^{r s}, r, s=(1, \cdots, 4)$. Construct then the $S O(4)$-connection and curvature $W^{r s} \equiv n^{s a} D n^{r a}, T=d W+W W$. Since one has $T^{r s}=n^{r a} n^{s b}\left(F^{a b}+D \hat{y}^{a} D \hat{y}^{b}\right)$ and $\varepsilon^{a_{1} \cdots a_{5}} \hat{y}^{a_{5}}=\varepsilon^{r_{1} \cdots r_{4}} n^{r_{1} a_{1}} \cdots n^{r_{4} a_{4}}$, it is immediately seen that

$$
K=\frac{1}{2} \chi(T),
$$

i.e. one-half of the $S O(4)$-Euler-form of the curvature $T^{20}$. This implies that $K$ has in general semi-integer integrals over closed four-manifolds which do not intersect M5. However, its integral over a four-sphere linking M5 equals unity, since the Eulercharacteristics of a four-sphere is two: this reflects clearly the fact that the $M 5$-brane carries one unit of magnetic charge.

\footnotetext{
${ }^{20}$ The frames $n^{a r}$ can not be glued together continuously all over the target-space. Even in a spacetime with trivial topology they carry necessarily a singularity along a seven-manifold $M_{7}$ whose boundary is $M 5$. This implies that $T$ is singular along $M_{7}$ while $\chi(T)$, being $S O(4)$-invariant i.e. independent of $n^{a r}$, is singular only on $M 5$. This means that we have $d \chi(T)=0$ in the complement of $M 5$, while in the whole target-space we have $d \chi(T)=2 J_{5}$.
} 


\section{Appendices}

\section{A Regularizations}

Instead of giving general formulae for the "real algebraic approximation mode" [11], we present here examples one for an even and one for an odd current, generalizations being straightforward.

First we approximate our $\delta$-like currents $J$ with smooth ones $J^{\varepsilon}$ that preserve $S O(n)$ invariance and depend algebraically on $F^{a b}, y^{a}$ and $D y^{a}$; for $n=5$ and $n=4$ we have

$$
\begin{aligned}
& J_{5}^{\varepsilon}=\frac{1}{4(2 \pi)^{3}} \frac{\varepsilon}{\varepsilon^{2}+y^{2}} \varepsilon^{a_{1} \cdots a_{5}} D y^{a_{1}}\left(F^{a_{2} a_{3}} F^{a_{4} a_{5}}+\frac{4}{3\left(\varepsilon^{2}+y^{2}\right)} F^{a_{2} a_{3}} D y^{a_{4}} D y^{a_{5}}\right. \\
& \left.+\frac{8}{15\left(\varepsilon^{2}+y^{2}\right)^{2}} D y^{a_{2}} D y^{a_{3}} D y^{a_{4}} D y^{a_{5}}\right) \text {, } \\
& J_{4}^{\varepsilon}=\frac{1}{8(2 \pi)^{2}} \frac{\varepsilon}{\sqrt{\varepsilon^{2}+y^{2}}} \varepsilon^{a_{1} \cdots a_{4}}\left(F^{a_{1} a_{2}} F^{a_{3} a_{4}}+\frac{2}{\varepsilon^{2}+y^{2}} F^{a_{1} a_{2}} D y^{a_{3}} D y^{a_{4}}\right. \\
& \left.+\frac{1}{\left(\varepsilon^{2}+y^{2}\right)^{2}} D y^{a_{1}} D y^{a_{2}} D y^{a_{3}} D y^{a_{4}}\right) \text {. }
\end{aligned}
$$

These currents satisfy

$$
\begin{aligned}
\lim _{\varepsilon \rightarrow 0} J^{\varepsilon} & =J \\
d J^{\varepsilon} & =0 \\
\int_{M_{n}} J^{\varepsilon} & =1,
\end{aligned}
$$

where the last integral is along an $n$-dimensional manifold at $\sigma^{i}=\sigma_{0}^{i}$. The currents $J^{\varepsilon}$ enjoy therefore the following properties: they are closed and invariant, they entail the same total charge as $J$ and they are regular at $M$ : we have indeed the pullback formulae

$$
\begin{aligned}
& \left.J_{4}^{\varepsilon}\right|_{M}=\chi_{4}(f) \\
& \left.J_{5}^{\varepsilon}\right|_{M}=0
\end{aligned}
$$

where $\chi(f)$ for a generic even $n$ is the $S O(n)$-Euler-form on $M$. These formulae represent, in particular, an explicit realization of the Thom-isomorphism [30]. Regularized Chernkernels satisfy by definition

$$
J^{\varepsilon}=d K^{\varepsilon}, \quad \lim _{\varepsilon \rightarrow 0} K^{\varepsilon}=K,
$$

and it is not difficult to find explicit expressions. For the even kernel one gets

$$
K_{4}^{\varepsilon}=\frac{1}{4(2 \pi)^{3}} \varepsilon^{a_{1} \cdots a_{5}} \hat{y}^{a_{1}}\left(f_{1} F^{a_{2} a_{3}} F^{a_{4} a_{5}}+f_{2} F^{a_{2} a_{3}} D \hat{y}^{a_{4}} D \hat{y}^{a_{5}}+f_{3} D \hat{y}^{a_{2}} D \hat{y}^{a_{3}} D \hat{y}^{a_{4}} D \hat{y}^{a_{5}}\right),
$$


with

$$
\begin{aligned}
& f_{1}=\arctan \frac{y}{\varepsilon} \\
& f_{2}=2\left(\arctan \frac{y}{\varepsilon}-\frac{\varepsilon y}{\varepsilon^{2}+y^{2}}\right) \\
& f_{3}=\arctan \frac{y}{\varepsilon}-\frac{\varepsilon y}{\varepsilon^{2}+y^{2}}-\frac{2}{3} \frac{\varepsilon y^{3}}{\left(\varepsilon^{2}+y^{2}\right)^{2}},
\end{aligned}
$$

and it is straightforward to see that for $\varepsilon \rightarrow 0$ one obtains (3.14) for $n=5$. For the odd kernel we get instead

$$
K_{3}^{\varepsilon}=\Omega_{3}^{\varepsilon}+\chi_{3}^{0}(A)
$$

where

$$
\Omega_{3}^{\varepsilon}=\frac{1}{2(4 \pi)^{2}} \varepsilon^{a_{1} \ldots a_{4}} \hat{y}^{a_{1}} D \hat{y}^{a_{2}}\left(g_{1} F^{a_{3} a_{4}}+g_{2} D \hat{y}^{a_{3}} D \hat{y}^{a_{4}}\right)
$$

with

$$
\begin{aligned}
& g_{1}=4\left(\frac{\varepsilon}{\sqrt{y^{2}+\varepsilon^{2}}}-1\right) \\
& g_{2}=\frac{8}{3}\left(\frac{\varepsilon^{3}+\frac{3}{2} \varepsilon y^{2}}{\left(y^{2}+\varepsilon^{2}\right)^{3 / 2}}-1\right) .
\end{aligned}
$$

For $\varepsilon \rightarrow 0 \Omega_{3}^{\varepsilon}$ reduces to (3.31). The regularized even kernel is invariant, as $K_{4}$, and the odd one maintains the transformation property of $K_{3}$ since $\Omega_{3}^{\varepsilon}$ is invariant. Both kernels are regular on $M$ and one gets

$$
\begin{aligned}
\left.K_{3}^{\varepsilon}\right|_{M} & =\chi_{3}^{0}(a) \\
\left.K_{4}^{\varepsilon}\right|_{M} & =0
\end{aligned}
$$

obviously in agreement with A.3 , A.4 .

To illustrate the use of these formulae we provide a simple alternative proof of (3.24). Considering the limits in the sense of distributions we can write

$$
d(Y K)=\lim _{\varepsilon \rightarrow 0} d\left(Y K^{\varepsilon}\right)=\lim _{\varepsilon \rightarrow 0}\left(d Y K^{\varepsilon}+Y J^{\varepsilon}\right),
$$

where we are allowed to use Leibnitz's rule since $K^{\varepsilon}$ is a regular distribution. The first term converges trivially to $d Y K$, while the second term is in the limit necessarily supported on $M$ (as $\varepsilon \rightarrow 0$ only terms with $y=0$ contribute, see (A.1) ). This means that

$$
\lim _{\varepsilon \rightarrow 0} Y J^{\varepsilon}=\mathcal{Y} J
$$

for some form $\mathcal{Y}$ which is defined on $M^{21}$. Moreover, since $Y$ as well as $J^{\varepsilon}$ are $S O(n)-$ invariant forms, also $\mathcal{Y}$ must be an invariant form on $M$; but since $\mathcal{Y}$ is an odd form it

\footnotetext{
${ }^{21}$ Notice that the existence of the $\operatorname{limit}_{\varepsilon \rightarrow 0} Y K^{\varepsilon}=Y K$ in the distributional sense ensures the existence of the $\operatorname{limit}_{\lim _{\varepsilon \rightarrow 0}} d\left(Y K^{\varepsilon}\right)$ in the distributional sense; the same property does in general not hold in linear spaces of functions, endowed with stronger topology.
} 
must necessarily vanish, because there are no invariant odd forms on $M$, made out of the $S O(n)$-connection $a^{a b}$.

Similarly one can show that for even kernels the product $K K$ is a closed form,

$$
d(K K)=\lim _{\varepsilon \rightarrow 0} d\left(K K^{\varepsilon}\right)=\lim _{\varepsilon \rightarrow 0}\left(J K^{\varepsilon}+K J^{\varepsilon}\right)=\lim _{\varepsilon \rightarrow 0} K J^{\varepsilon}
$$

where the last equality follows from (A.12). As above one has $\lim _{\varepsilon \rightarrow 0} K J^{\varepsilon}=\mathcal{K} J$, for some invariant even form $\mathcal{K}$ which must be also odd under parity of the normal bundle i.e. proportional to $\varepsilon^{a_{1} \cdots a_{n}}$, as is $K$. But since $n$ is odd no such form exists, and hence $\mathcal{K}=0$.

\section{B Existence and construction of the three-form $X$}

We begin giving the explicit expression of the $S O(5)$-kernel

$$
K=\frac{1}{4(4 \pi)^{2}} \varepsilon^{a_{1} \cdots a_{5}} \hat{y}^{a_{1}}\left(F^{a_{2} a_{3}}+D \hat{y}^{a_{2}} D \hat{y}^{a_{3}}\right)\left(F^{a_{4} a_{5}}+D \hat{y}^{a_{4}} D \hat{y}^{a_{5}}\right),
$$

and recalling the definition of the Euler-form, the associated Chern-Simons form and its descent, for a generic $S O(4)$-bundle with connection $W$ and curvature $T=d W+W W$,

$$
\begin{aligned}
\chi(T) & =\frac{1}{2(4 \pi)^{2}} \varepsilon^{r_{1} \cdots r_{4}} T^{r_{1} r_{2}} T^{r_{3} r_{4}}, \\
\chi^{0}(W) & =\frac{1}{2(4 \pi)^{2}} \varepsilon^{r_{1} \ldots r_{4}}\left(W^{r_{1} r_{2}} d W^{r_{3} r_{4}}+\frac{2}{3} W^{r_{1} r_{2}}(W W)^{r_{3} r_{4}}\right), \\
\chi^{1}(W) & =\frac{1}{2(4 \pi)^{2}} \varepsilon^{r_{1} \ldots r_{4}} L^{r_{1} r_{2}} d W^{r_{3} r_{4}}, \\
\chi(T) & =d \chi^{0}(W), \quad \delta \chi^{0}(W)=d \chi^{1}(W), \quad \delta W^{r s}=D L^{r s} .
\end{aligned}
$$

We are searching for a form $X$ on $M_{3}$ such that

$$
K J_{8}+\frac{1}{2} \chi_{b}^{0} J_{9}=d\left(X J_{8}\right)
$$

where $\chi_{b}^{0}(w)$ is the Chern-Simons form of the Euler-form, with $S O_{b}(4)$-connection $w^{r s}$ on $M_{2}$. Since $K J_{8}=\left.K\right|_{M_{3}} J_{8}$, what appears really in this formula is the pullback of $K$ on $M_{3}$, and we will now show that the pullback form is a closed form, more precisely that

$$
\left.K\right|_{M_{3}}=d X
$$

where $X$ is the form we are searching for. In $\left.K\right|_{M_{3}}$ all objects of (B.1) are evaluated on $M_{3}$. If we parametrize $M_{3}$ by $x^{\mu}(\sigma)$ we get in particular $u^{a}(\sigma) \equiv \hat{y}^{a}(x(\sigma))$, which determines in each point of $M_{3}$ a particular direction. This allows to define on $M_{3}$ the reduction - along $u^{a}$ - of the $S O(5)$-connection $A^{a b}$ down to an $S O(4)$-connection $W^{r s}(\sigma)$. The reduction procedure is canonical: define on $M_{3}$ an $S O(4)$-basis $n^{r a}$ normal to $u^{a}, r=(1, \cdots, 4)$,

$$
n^{r a} u^{a}=0, \quad n^{r a} n^{s a}=\delta^{r s}, \quad u^{a} u^{a}=1
$$


then the reduced connection and curvature are given by

$$
W^{r s}=n^{s a} D n^{r a}, \quad T^{r s}=d W^{r s}+W^{r t} W^{t s} .
$$

With these definitions it is straightforward to show that one has the identities

$$
T^{r s}=n^{r a} n^{s b}\left(\left.F^{a b}\right|_{M_{3}}+D u^{a} D u^{b}\right), \quad \varepsilon^{a_{1} \cdots a_{5}} u^{a_{5}}=\varepsilon^{r_{1} \cdots r_{4}} n^{r_{1} a_{1}} \cdots n^{r_{4} a_{4}} .
$$

Using them in the restriction of (B.1 $)$ to $M_{3}$, one sees immediately that this restriction amounts to the Euler-form of $T$, apart from a factor $1 / 2$,

$$
\left.K\right|_{M_{3}}=\frac{1}{2} \chi(T)
$$

The three-form $X$ is then simply the Chern-Simons form of $W$,

$$
X=\frac{1}{2} \chi^{0}(W)
$$

To prove that this $X$ satisfies indeed $(\underline{B} .2)$ it remains to show that the restriction of $W^{r s}$ from $M_{3}$ to $M_{2}$ coincides with the $S O_{b}(4)$-connection $w^{r s}$. This can be seen as follows. Since the $y^{a}$ are normal coordinates on $M_{6}$ and $u^{a}=\left.\hat{y}^{a}\right|_{M_{3}}$, we have that the vector $\left.\widetilde{u}^{a} \equiv u^{a}\right|_{M_{2}}$ coincides with the (normalized) component normal to $M_{6}$, of the unique vector on $M_{2}$ which is tangent to $M_{3}$ and normal to $M_{2}$. But by definition (see section 5.1) $S O_{b}(4)$ is the subgroup of $S O(5)$ on $M_{2}$ which leaves $\widetilde{u}^{a}$ invariant. This means, by construction, that $\left.W^{r s}\right|_{M_{2}}=w^{r s}$.

From the explicit construction of $X$ one can deduce its transformation properties under $Q$-transformations and $S O_{b}(4)$-transformations respectively, checking thus (5.18) and (5.19):

$$
\begin{aligned}
\Delta X & =\left.Q\right|_{M_{3}}+d \phi, \\
\delta X & =\frac{1}{2} d \chi^{1}(W),
\end{aligned}
$$

where $\phi$ is some two-form on $M_{3}$ with $\left.\phi\right|_{M_{2}}=0$, and clearly $\left.\chi^{1}(W)\right|_{M_{2}}=\chi_{b}^{1}$.

\section{PST-invariance}

The main work one has to accomplish is to compute the variation of $S$ under generic variations of the fields $B_{3}, B_{6}, \widehat{b}$ and $C$. The result can be cast in the form

$$
\begin{aligned}
\delta S= & \int_{M_{11}}\left[\left(d\left(V f_{6}\right)+V f_{3} H_{4}-g v(\mathcal{H}-\mathcal{W}) J_{5}\right)\left(\delta B_{3}+d \delta \widehat{b}\right)\right. \\
& -d\left(V f_{3}\right)\left(\delta B_{6}+\frac{1}{2} B_{3} \delta B_{3}+g K \delta \hat{b}\right) \\
& \left.+d C d\left(\frac{g}{2 \partial c \partial c}(\mathcal{H}-\mathcal{W})(\mathcal{H}-\mathcal{W}) J_{5}-\frac{1}{\partial C \partial C} f_{3} f_{6}\right) \delta C\right] .
\end{aligned}
$$


To arrive at this formula one has to use (6.15) and some standard relations like $i_{V} *=* V$ and $* i_{V}=V *$; but, most importantly, one has to use the identity for four-forms

$$
\mathcal{H} \mathcal{H}=\mathcal{W W}
$$

which holds because $\mathcal{W}$ is the functional derivative of the Born-Infeld lagrangian. It is indeed the qualifying property for all allowed lagrangians, i.e. those which give rise to a consistent (Lorentz-invariant) dynamics for a (self-interacting) chiral boson in six dimensions.

The equations of motion for $B_{6}, B_{3}, \widehat{b}$ and $C$ can be read directly from $\delta S$ and are given respectively by,

$$
\begin{array}{rlrl}
d\left(V f_{3}\right) & =0 & \text { (C.1) } \\
d\left(V f_{6}\right) & =g v(\mathcal{H}-\mathcal{W}) J_{5}-V f_{3} H_{4}(\mathrm{C} .2) \\
g d(v(\mathcal{H}-\mathcal{W})) J_{5} & =-d\left(V f_{3} H_{4}\right) & \text { (C.3) } \\
d C d\left(\frac{g}{2 \partial c \partial c}(\mathcal{H}-\mathcal{W})(\mathcal{H}-\mathcal{W}) J_{5}-\frac{1}{\partial C \partial C} f_{3} f_{6}\right) & =0 .
\end{array}
$$

One sees that the $\widehat{b}$-equation is supported on $M_{6}$, because the r.h.s. of (C.3) is proportional to $J_{5}$, thanks to (C.1). This is consistent with the fact that the extension $\widehat{b}$ is a pure gauge degree of freedom. It is also immediate to see that the $\widehat{b}$-equation is a consequence of the $B_{3}$-equation, and that the $C$-equation is a consequence of the other three equations.

Eventually, proving invariance of $S$ under the PST-transformations (6.19)-(6.23) given the above form of $\delta S-$ is a simple exercise.

Acknowledgements. The authors thank M. Cariglia and M. Tonin for useful discussions. This work is supported in part by the European Community's Human Potential Programme under contract HPRN-CT-2000-00131 Quantum Spacetime.

\section{References}

[1] S. Chern, Ann. Math. 45 (1944) 747; Ann. Math. 46 (1945) 674.

[2] D. Freed, J.A. Harvey, R. Minasian and G. Moore, Adv. Theor. Math. Phys. 2 (1998) 601; J.A. Harvey and O. Ruchayskiy, JHEP 06 (2001) 044.

[3] K. Becker and M. Becker, Nucl. Phys. B577 (2000) 156.

[4] A. Boyarsky and B. Kulik, Phys. Lett. B516 (2001) 171.

[5] A. Boyarsky, J.A. Harvey and O. Ruchayskiy, Ann. Phys. 301 (2002) 1.

[6] Ph. Brax and J. Mourad, Phys. Lett. B416 (1998) 295. 
[7] K. Lechner, P.A. Marchetti and M. Tonin, Phys. Lett. B524 (2002) 199.

[8] G. de Rham, Differentiable manifolds. Forms, Currents, Harmonic Forms, Springer Verlag 1984.

[9] K. Lechner and P.A. Marchetti, JHEP 01 (2001) 003.

[10] K. Lechner and P.A. Marchetti, Nucl. Phys. B (Proc. Suppl.) 102 \& 103 (2001) 94.

[11] F.R. Harvey and H.B. Lawson Jr., A theory of characteristic currents associated with a singular connection, Asterisque 213 (1993), Sociètè Mathèmatique de France.

[12] R. Bott and A.S. Cattaneo, J. Diff. Geom. 48 (1998) 91.

[13] K. Intriligator, Nucl. Phys. B581 (2000) 257.

[14] M. Cariglia and K. Lechner, Phys. Rev. D66 045003 (2002).

[15] P. Pasti, D. Sorokin and M. Tonin, Phys. Rev. D55 (1997) 6292; R. Medina and N. Berkovits, Phys. Rev. D56 (1997) 6388.

[16] A. Strominger, Phys. Lett. B383 (1996) 44.

[17] P.K. Townsend, Phys. Lett. B373 (1996) 68.

[18] M.J. Duff, J.T. Liu and R. Minasian, Nucl. Phys. B452 (1995) 261.

[19] J. Schwarz, Phys. Lett. B367 (1996) 97; S. de Alwis, Phys. Lett. B388 (1996) 291.

[20] E. Witten, J. Geom. Phys. 22 (1997) 103.

[21] P. Pasti, D. Sorokin and M. Tonin, Phys. Lett. B398 (1997) 41.

[22] I. Bandos, N. Berkovits and D. Sorokin, Nucl. Phys. B522 (1998) 214.

[23] I. Bandos, K. Lechner, A. Nurmagambetov, P. Pasti, D. Sorokin and M. Tonin, Phys. Rev. Lett. 78 (1997) 4332.

[24] M. Aganagic, J. Park, C. Popescu and J.H. Schwarz, Nucl. Phys. B496 (1997) 191.

[25] S.P. de Alwis, Phys. Rev. D56 (1997) 7963.

[26] J. Kalkkinen and K.S. Stelle, hep-th/0212081.

[27] X. Bekaert and A. Gomberoff, hep-th/0212099.

[28] E. Witten, J. Geom. Phys. 22 (1997) 1.

[29] E. Witten, JHEP, 0005 (2000) 031.

[30] R. Bott and L.W. Tu, Differential forms in algebraic geometry, Springer Verlag 1978. 